\title{
40th anniversary of introduction of expanded immunization program (EPI): a literature review of introduction of new vaccines for routine childhood immunization in sub-saharan africa
}

\author{
Volume I Issue I - 2015 \\ Jean Paul Uwizihiwe, Hans Bock \\ University of Siena, Italy
}

\section{Abstract}

Background \& objective: The year 2014, marks an important public health anniversary. It is exactly 40years ago that the World Health Organization (WHO) established the Expand Program Immunization (EPI). Since its launch, countries in the region of sub-Saharan Africa (SSA) have been found to be behind regarding new vaccine introduction (NVI) such as HepB, HibCV, PCV, RotaV and HPV. These vaccines have been developed in the era post EPI establishment and are currently recommended by WHO for routinely immunization for all. The objective of this thesis is to give an update of the status of these new vaccines introduced for routine childhood immunization in SSA since the establishment of EPI in 1974 and to identify their challenges and opportunities.

Methods and findings: A systematic review including a grey literature search was conducted to identify papers reporting about the introduction of new vaccines in the routine immunization programs of SSA countries. Different databases (PubMed, ISI Knowledge Center, and AIM) and records from WHO, UNICEF, MoH, NGO and other agencies working on immunization were searched by using keywords and were limited to the articles or reports published in the last five years. Inclusion and exclusion criteria were applied to find the most relevant articles or reports for this review. The search yielded 1087 papers dating from May $31^{\text {st }}$ May 2009 to May $31^{\text {st }}$ 2014of which $44(4 \%)$ were found to be relevant and included in the review analysis. We found that half the number of deaths in children under the age of five worldwide due to new VPDs is derived from the region of SSA. However, in the recent decade, and with the support of the several donors, the majority of SSA countries have made remarkable progress in introducing new vaccines into their NIP. HepB and $\mathrm{HibCV}$ vaccines have been added to all the immunization programsof 48 countries. The introduction of PCV and rotavirus vaccines has reached $66 \%$ and $41 \%$ of countries respectively. PCV-13 and monovalent rotavirus vaccines are the ones most widely used in immunization programs across SSA countries. For HPV vaccination Rwanda and South Africa completed the roll-out at national level through a schoolgirl based approach, and several other countries in SSA have started HPV demonstration projects. The implementation of school based vaccination programs in SSA countries given still lower school enrolment and attendance constitutes however a challenge. The WHO recommended immunization schedule of $6,10,14$ weeks for infant vaccinations has been adopted by majority of SSA countries. The routine administration of birth dose monovalent HepB vaccine is currently only given in seven countries in SSA and poses a challenge as many home deliveries still occur in SSA limited access for skilled health-care providers at the time of childbirth
Correspondence: Jean Paul Uwizihiwe, University of Siena, Via Banchi di Sotto, 55, 53100 Siena SI, Italy, Tel +39 0577 232 I I I, Email uwizihiwejp@gmail.com

Received: June 01, 2015 | Published: July 02, 2015
The GAVI Alliance has provided an opportunity for many SSA countries to procure new vaccines. Good governance is the most important factor for donors to determine the ability of SSA countries to introduce new vaccines. Countries in SSA with proactive planning benefit regarding scaling up the systems of cold chain and vaccine logistics, improvement of surveillance and reporting procedures as well as the training of the health care workforce. The launch of new vaccine in EPI has become a political opportunity for African top officials and first ladies. The immunization programs in SSA however still remain heavily donor dependent. The only two SSA countries planned for GAVI graduation for self-sufficiency are Angola and Congo. There is still no clear African ownership initiative recognizable in order to overcome external donor dependence in the region. Learning from the success story in Latin America lead by PAHO is a crucial need for the countries and region of SSA.

Conclusion: Although far from having achieved its high aiming goals, the EPI in SSA has come a long way and made a good progress more recently in strengthening the delivery of immunization services and to accelerate efforts to achieve the control of the VPDs in the region of SSA over the last 40years. With the 2015 deadline for the MDGs approaching, it is necessary for Africa to critically assess its position with regards to the NVI, take ownership of the entire regional, sub-regional and country-specific problems, and develop more precise strategies to overcome the challenges identified as well as start looking beyond the MDGs for a better future for the people in Africa.

Keywords: immunization; world health organization, SSA, PAHO, hepatitis $B$; new vaccines; UNICEF, GAVI, expand program immunization; rotavirus vaccines, HibC, PCV, RotaV, Africa
Abbreviations: WHO, world health organization; SSA, sub-saharan africa; NVI, new vaccine introduction; EPI, expand program immunization; WHA, world health assembly; Vpds, vaccine preventable disease; BCG, bacillus calmette-guérin; GAVI, global alliance for vaccines and immunization; WEF, world economic forum; UNICEF, united nations children's fund; Hibcv, haemophilus influenza type B conjugate vaccines; Hepb, hepatitis B vaccines; PCV, PATH, pneumococcal conjugate vaccines; Rotav, rotavirus vaccines; HPV, human papilloma virus vaccines; rotavirus vaccine access and de- livery ; IVAC, international vaccine access center; VIMS, vaccine information management system: CMYP, comprehensive multiyear plan; PIE, post-introduction evaluations; THECAP, healthcare expenditure per capita; GHECAP, government healthcare expenditure per capita; HEGDP, healthcare expenditure as percent of the GDP

\section{Background}

After clean water, immunization is considered to be the second most important basic human right. It is the most effective public health 
intervention in the world for saving lives and promoting good health. The World Health Organization (WHO) defines immunization as a process whereby a person is made immune or resistant to an infectious disease, typically by the administration of a vaccine. ${ }^{2}$ To-date, the list of vaccine preventable disease (VPDs) has been extended to $25 .{ }^{3}$ These vaccines are against anthrax, measles, rubella, cholera, meningococcal disease, influenza, diphtheria, mumps, tetanus, hepatitis A, pertussis, tuberculosis, hepatitis B, pneumococcal disease, typhoid fever, hepatitis E, poliomyelitis, tick-born encephalitis, haemophilus influenza type b, rabies, varicella and herpes zoster (shingles), human papillomavirus, rotavirus gastro-enteritis, yellow fever and Japanese encephalitis. During the $20^{\text {th }}$ century, vaccines have been able to eradicate and eliminate several childhood diseases that used to kill millions of children. Significant contributions from vaccination include the eradication of smallpox, the almost complete elimination of poliomyelitis, and a decrease of many others infectious diseases such as diphtheria, tetanus, pertussis, measles, mumps and rubella. ${ }^{4}$ The 20th century has been marked by great successes at developing vaccines for many acute infectious diseases. ${ }^{5}$ In the last two decades, developing countriesshown commitment and implemented strategies and programs to avail these vaccines to their population. WHO had established the Expanded Program of Immunization (EPI) to scale up immunization programmes throughout the world and served as a basis for all the nations worldwide to initiate their immunization known as national immunization program (NIP). ${ }^{6}$ The NIP is the organizational component of Ministry of Health charged with preventing disease, disability, and death from VPDs in children and adults. ${ }^{7}$ The NIP is used interchangeably with the EPI that originally focused on preventing VDPs in children. On the core of this thesis is the introduction of new vaccines in Sub-Saharan Africa (SSA) in the period following the establishment of EPI.

\section{History of expanded program immunization}

The year 2014, it is exactly forty years ago that the WHO established the EPI through its resolutionWHA27.57 adopted in the World Health Assembly (WHA) in May 1974.The resolution urges countries to build on the success of the global smallpox eradication programme, and it wishes to ensure that all children in all countries benefit from life-saving vaccines. ${ }^{8,9}$ The EPI has been the catalyzer to fight the VPDs and it lays the foundation for many other future immunization initiatives. The first six VPDs targeted by the EPI were diphtheria, whooping cough, tetanus, measles, poliomyelitis and tuberculosis. When the EPI was initiated in 1974, less than 5percent of children in developing countries were receiving a third dose of diphtheria-tetanus-pertussis (DTP-3) and poliomyelitis vaccines in their first year of life..$^{10}$ In the period following the establishment of EPI, WHO set a goal to make immunization against those six basic diseases available to every child in the world by 1990 . The general program policies, including this EPI goal were adopted in May 1977 and approved in the resolution WHA30.53. ${ }^{11,12}$

In 1984, ten years after the establishment of EPI, the WHO established a standardized vaccination schedule for the original EPI vaccines: Bacillus Calmette-Guérin (BCG) one dose at birth, DTP-3 doses-booster 1 to 6years of age, oral polio 3 doses, with DTP, and measles 2 doses. During this period, most of the developing countries including the countries in the region of SSA started prioritizing the inclusion of the six original EPI vaccines in their health programs. The ambition for universal childhood immunization in the 1980s and the global effort to eradicate polioas well as the increased knowledge of the immunologic factors of disease led to new vaccines being developed and added to the EPI's list of recommended vaccines: Hepatitis B (HepB), Yellow Fever in countries endemic for the disease, and Haemophilus influenzae type $b$ conjugate vaccine (HibCV)in countries with high burden of disease. ${ }^{13}$ The HepB vaccination is available in different formulations and in combination with other vaccines (Hib, DTaP, IPV and HAV). The type of HepB vaccines currently being used is the recombinant vaccine. The HepB recombinant vaccines resulted from a second generation of genetically engineered (or DNA recombinant) vaccines that was carried out in 1986. ${ }^{14}$ The first HibCV was developed and licensed in USA in $1987 .{ }^{15}$ In May 1995 following the first basic WHA resolution on global immunization program, the United Nation in its WHA 48.12 resolution requested WHO to facilitate provision in the near future of means for prevention of acute respiratory infections, such as Hib vaccine and a conjugate pneumococcal vaccine for children in developing countries. In January2000, the Global Alliance for Vaccines and Immunization (GAVI) was created through an international public and private coalition at the World Economic Forum (WEF) in Davos, Switzerland. Initially, with the main financial contribution from the Bill \& Melinda Gates Foundation, the GAVI has the sole purpose of improving child health in the poorest countries by extending the reach of the EPI. ${ }^{16}$ Although global immunization coverage has increased during the past decade to levels of around 78 percent for DTP-3, the region of SSA has consistently ranking behind, reaching only 69 percent DTP-3 coverage by $2004 .{ }^{17}$ In response to these challenges in global immunization, in 2006 WHO and UNICEF developed the Global Immunization Vision and Strategy (GIVS). The GIVS is the first ever ten-year framework aimed at controlling morbidity and mortality from vaccine-preventable diseases and helping countries to immunize more people, from infants to seniors, with a greater range of vaccines. The main goal of GIVS is to reduce illness and death due to vaccine-preventable diseases by at least two-thirds by 2015 or earlier. ${ }^{18}$ In May 2012, the WHA in its resolution WHA65.17 adopted the Global Vaccine Action Plan (GVAP). The GVAP is a ten year framework (2011-2020) which aims to achieve the Decade of Vaccines vision by delivering universal access to immunization. ${ }^{19,20}$

\section{Initiation of expanded program immunization in Africa}

According to the WHO report on establishment of EPI in developing countries, the first seminar on expansion of the use of immunization in developing countries was held in Africa, in Kumasi in Ghana, from 12 to 19 November 1974. The seven day seminar gathered participants of eleven different countries of SSA (Ghana, Ethiopia, Nigeria, Kenya, Liberia, Sierra Leone, Somalia, Sudan, Uganda, United Republic of Tanzania and Zambia) and representatives of various organizations (WHO, UNICEF, SIDA and OCCGE). Subsequently, the Kumasi seminar followed the 27th WHA resolution asking WHO to assist the developing countries to bring under control the diseases susceptible to immunization..$^{21,22}$ The seminar clearly defined existing problems in the participating countries, and they have been encouraged to overcome them first by themselves and only then, looking for external support when a gap was clearly defined. The Kumasi seminar recognized that most of the countries could contribute greatly to expansion of programmes by making better use of their available national resources. Finally, the seminar recognized the great importance of the use of available vaccines with proven efficacy for a rapid control of the seven diseases- measles, poliomyelitis, pertussis, tuberculosis, tetanus, diphtheria and smallpox. The EPI in the African region was launched by most of the countries in the late1970's and by mid 1980sall countries had established NIP. Following the evaluation made in 1985, which showed that the immunization coverage was in general lower for all six original antigens in African countries in comparison of other countries, African member states agreed to 
accelerate the programme by adopting a resolution declaring 1986 the "African Immunization Year» and pursued implementation of various accelerated efforts until 1990. During the acceleration phase, there been a strong political commitment with the involvement of top national officials in launching the immunization campaign in many countries in SSA. ${ }^{23}$

Over the last forty years, there has been significant improvement in EPI in Africa. These progresses have been made in improving especially the use of the routine vaccines. As May 2014, WHO recommends at least 12 antigens to be included into immunization program of all countries as use of all. ${ }^{24}$ DTP- 3 coverage is widely recognized as a good indicator of the strength of routine immunization services. ${ }^{17}$ According to WHO and the United Nations children's Fund (UNICEF) coverage estimates, the number of countries with national DTP-3 coverage of at least 80 percent increased from one (2 percent) in 1980, through 22 (42 percent) in 1990 and 17 (32 percent ) in 2000, to 35 (67 percent) in $2010 .{ }^{25}$ Although the overall routine expanded immunization in Africa has improved in coverage, it still faces some major challenges such as cold chain management, implementation of use of auto-disable syringes, adequate safety and waste disposal measures. ${ }^{26}$ Furthermore, the improvement of immunization spending in most African countries has predominantly been due to donor funds. ${ }^{17}$ Against this backdrop of challenges, countries in the region of SSA have been continuously pointed out as being behind in the introduction of new vaccines into their routine immunization programs.

A "new vaccines introduction (NVI)" can mean the addition to an immunization programme of a vaccine against a disease not previously covered by an immunization programme (e.g. rotavirus or HPV vaccines). It can also describe the introduction of a new combination vaccine (e.g. DTP-HepB-Hib) replacing previous individual vaccines for the various component antigens, or a new product formulation of a vaccine already in the programme (e.g. a liquid vaccine replacing a lyophilized vaccine), or a vaccine that uses a new route of administration in place of a currently-used vaccine (e.g. an injectable vaccine replacing an oral vaccine).$^{27}$ In this review, the term "new vaccines" is used as a new antigen or a new combination vaccine that demands changes in the activities of an immunization programme for successful and widespread delivery to a population. Our review will only focus on the five new vaccines beyond the six EPI original vaccines (so called traditional or old EPI vaccines) that have been developed in the post era of EPI and recommended by WHO for routinely immunization for all. These include hepatitis B (HepB) vaccines; Haemophilus Influenza type B conjugate vaccines (HibCV), pneumococcal conjugate vaccines (PCV) and rotavirus vaccines (RotaV) and human papilloma virus vaccines (HPV). Note that Rubella vaccine is currently among the 12 vaccines routinely recommended by WHO for the use of all. Although the inclusion of rubella into EPI occurred the year after establishment of EPI, the discovery of rubella vaccine happened before $1974 .{ }^{28,29}$

\section{Objectives}

The overall purpose of this thesis project was to give an overview of the new vaccines introduced for routine childhood immunization in sub-Saharan Africa since the establishment of Expanded Immunization Program forty years ago. The study has three specific objectives:

i. To provide an update on the progress of the introduction of new vaccines for routine childhood immunization introduced in the immunization programs in sub-Saharan Africa via a review of published and unpublished data/ information within the last five years. ii. To produce an updated report on trends and challenges related to the introduction of new vaccines for routine childhood immunization in the countries of sub-Saharan Africa via a review of published and unpublished data/ information within the last five years and

iii. To provide an updated report on the opportunities and benefits which the introduction of new vaccines for routine childhood immunization into EPI in countries in sub-Saharan Africa could further have in the future via a review of published and unpublished data/ information within the last five years.

\section{Methods}

As defined in the previous section, New Vaccines Introduction (NVI) is the introduction of any vaccine beyond the original six EPI vaccines against diphtheria, pertussis, tetanus, measles, poliomyelitis, and tuberculosis. Our review has focused on all new vaccines that have been developed in the era following the establishment of EPI and are currently recommended by WHO for routine use. The range of methods used to give an overview of the new vaccines for routine childhood immunization for the last forty years - post period of establishment of EPI in SSA included both a review of published literature and a review of grey literature. The grey literature review consisted of written material or information produced on all levels of government, academics, business and industry in electronic and print formats not controlled by commercial publishing i.e. where publishing is not the primary activity of the producing body (for instance reports).

\section{Published literature review}

A systematic review of published literature was conducted to identify articles reporting on introduction of new vaccines in the routine national immunization program (i.e. public sector) of countries in the region of SSA. Three publication databases (PubMed, African Index Medicus and ISI Knowledge of Center) were searched for the published literature review, using keywords terms(the combination of free text and MESH terms)encompassing EPI, routine immunization program, new vaccines introduction, challenges, opportunities, impact, under-five mortality, diseases burden, vaccines preventable diseases, names of African countries and sub-Sahara Africa. The search has been completed on June $30^{\text {th }} 2014$ and limited to the articles or reports published in the last five years (May31 2009 to May3 $\left.1^{\text {st }} 2014\right)$ to ensure the review of the most recent papers.

\section{Grey literature review}

A systematic search of the grey literature included documents that were not peer-reviewed or published commercially, and were written for the period of May $31^{\text {st } 2009}$ to May 31 2014 . ISI Knowledge Center and WHO office data bases. We reviewed national immunization programmes of all countries of SSA region. We carried out a literature review of official documents produced by ministries of health $(\mathrm{MoH})$ of countries in the region of SSA, UNICEF and WHO. In addition, websites of agencies working on immunization and NVI were searched, and additional documents were identified through networking.

\section{Inclusion and exclusion criteria}

The inclusion criteria for an article to be used in this review are:

i. Study/review/report must be relevant to expanded program on immunization to the region of SSA and/or to any country in the region of SSA. 
ii. Study/review/report must be relevant to the introduction of the five vaccines (hepatitis type B, Haemophilus Influenzae type b, human papilloma virus, pneumococcal conjugate and rotavirus) into NIP of countries of SSA.

iii. Date of publication must be in the period of May $31^{\text {st }} 2009$ to May $31^{\text {st }} 2014$.

iv. Results were restricted to original research articles, papers or reports written in English. Conversely, the exclusion criteria are those not complying with the above requirements.

\section{Results}

\section{Overview of articles selected}

The initial electronic search resulted in a total of 459 English citations including 272 PubMed,91ISI knowledge center, 52 African Index Medicus (AIM) and44from different databases: WHO, UNICEF, $\mathrm{MoH}$, NGOs, etc. After applying manual de-duplication and the exclusion criteria on the basis of titles and abstract, 436 (95percent) were eliminated. The remaining yield of 23 (5percent) are articles, papers or reports that were obtained and included in the review.

\begin{tabular}{|c|c|c|}
\hline & Name of The First Author and Reference & Source and Year of Publication \\
\hline 1. & Binagwaho et al. (41) & WHо Bulletin, 2013 \\
\hline 2. & Bingham et al.(103) & Archives of pediatrics \& adolescent medicine, 2009 \\
\hline 3. & Burchett et al. (78) & Health Policy and Planning, 2012 \\
\hline 4. & Candotti et al. (111) & The lournal of general virology, 2009 \\
\hline 5. & Chan et al. (126) & The Lancet, 2014 \\
\hline 6. & Duclos et al. (121) & BMC international health and human rights, 2012 \\
\hline 7. & Glatman-Freedman et al. (84) & Public Library of Science, 2013 \\
\hline 8. & Gordon et al. (74) & Health Policy and Planning, 2012 \\
\hline 9. & Gotberg et al. (15) & Journal of Vaccine, 2011 \\
\hline 10. & Hajjeh et al. (51) & Philosophical Transactions B, 2011 \\
\hline 11. & Howell et al. (116) & Journal of viral hepatitis, 2014 \\
\hline 12. & JHSPH-IVAC (31) & VIMS Report,2014 \\
\hline 13. & Kramvis et al. (113) & Journal of Vaccine, 2010 \\
\hline 14. & Machingaidze et al. (26) & Public Library of Science, 2013 \\
\hline 15. & Madhi et al. (33) & South Africa Medical Journal, 2014 \\
\hline 16. & Madhi et al. (61) & NEIM, 2010 \\
\hline 17. & Mantel et al. (73) & Health Policy and Planning, 2012 \\
\hline 18. & MoH in SSA (CMYP) (30) & WHO Country Planning Cycle, 2014 \\
\hline 19. & РATH (33) & Country Introduction Interactive Spreadsheet, 2014 \\
\hline 20. & Saxenian et al. (79) & Health Policy and Planning 2014 \\
\hline 21. & Shearer et al. (83) & PLOS medicine, 2010 \\
\hline 22. & Tagbo et al. (105) & The Pediatric infectious disease journal, 2014 \\
\hline 23. & พно (45) & WHO VDP global summary, 2014 \\
\hline
\end{tabular}

The background information for the included articles or reports is displayed on Table 1.

\section{Progress of the introduction of new vaccines}

The status of new vaccine introduction in SSA: The Table 2 displays the status (May 2014) of New Vaccine Introduction (NVI) in the National Immunization Program (NIP) (i.e. public sector) in each country of SSA, by type of vaccines and year of vaccine introduction. The type of vaccine in use and the year of introduction of each of the vaccines presented in table 2 have been abstracted and crossed checked in different sources such as to the WHO country planning cycle database,$^{30}$ different official documentation on immunization such as comprehensive multiyear plan (CMYP) of each country in SSA. In order to complete this table with updated information, introduction years was gathered from.

i. The Vaccine Information Management System (VIMS) database at Johns Hopkins Bloomberg School of Public Health's International Vaccine Access Center (IVAC) ${ }^{31}$ which collects introduction data from publicly available reports and

ii. The Rotavirus Vaccine Access and Delivery (PATH) country introduction report. ${ }^{32}$

Recombinant HepB, HibCV and combination vaccines: As May 2014, we found that all countries in the region of SSA had introduced both $\mathrm{HepB}$ and HibCV vaccines (48 out 48 countries, 100 percent). The Gambia was the first country on the continent to introduce the HepB vaccine in 1990 and Hib vaccine in 1997 long before the GAVI era. In the period of 1990s, other countries (Mauritius, Seychelles, South Africa and Swaziland) have completed the roll out of HepB vaccines in their NIP. During the last decade, most of the countries in the region of SSA have shifted to the use of combination vaccines. The new combination vaccines which contains five antigens Diphtheria, Tetanus, Pertussis, Inactivated Polio Vaccine and Haemophilus influenzae type b (DTaP-IPV-Hib known as Pentacel and not yet prequalified by WHO) is only used in South Africa and was introduced into their NIP in 2009.The Gambia (1997) and South Africa (1999) were the two first countries to introduce Hib vaccine into their NIP. With the support of GAVI, most countries in the region have changed to the use of pentavalent. Kenya was among the first countries to receive financial support from GAVI, and pentavalent vaccine was introduced nationwide in November 2001. The majority of the countries in the region added pentavalent vaccine in the period of 2005-2009 while they were introducing the Hib vaccine for the first time. Somalia and Equatorial Guinea became the two last countries in the region to introduce both vaccines (HepB and Hib) in form of pentavalent, respectively in April 2013 and Sept 2013. Pneumococcal vaccine introduction: South Africa became the first country in SSA to introduce PCV in April 2009. ${ }^{33}$

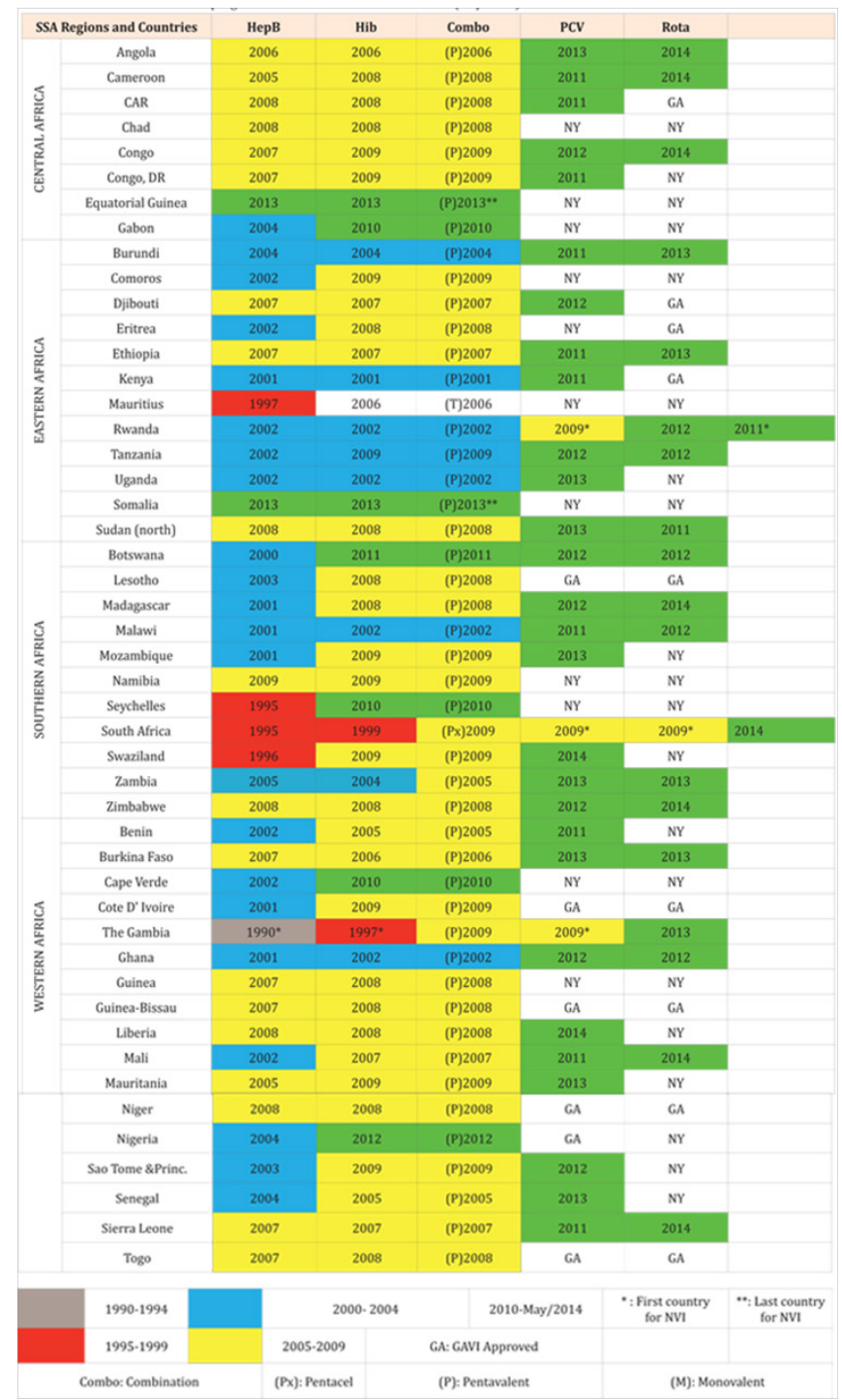


In July 2009, Rwanda introduced the PCV as the first GAVI-eligible country and one month later The Gambia followed. ${ }^{31}$ As of March 2014 , the rollout of pneumococcal vaccines has been completed in the 31 countries (66percent ) of SSA: Angola, Benin, Botswana, Burkina Faso, Burundi, Cameroon, Djibouti, CAR, Congo, DRC, Ethiopia, Ghana, The Gambia, Kenya, Liberia, Madagascar, Malawi, Mali, Mauritania, Mozambique, Rwanda, Senegal, Sierra Leone, Sudan, South Africa, Swaziland, Tanzania, Uganda, Zambia and Zimbabwe. The completion of PCV in the majority of these countries has been done with GAVI support from 2011 to 2013. According to the VIMS, GAVI has already approved the application for PCV introduction in six countries in the region: Côte d'Ivoire, Lesotho, Guinea Bissau, Niger, Nigeria and Togo, ${ }^{34}$ We found that 12 (25percent) countries of SSA have not yet introduced the PCV in their EPI program, these are Chad, Equatorial Guinea, Gabon, Comoros, Eritrea, Mauritius, Somalia, Namibia, Seychelles, Cape Verde and Guinea.

Rotavirus vaccines introduction: Compared to the previous described vaccine (PCV), there have been a slow progress in the introduction of Rota vaccine into NIP of countries in SSA. As of April 2014, a total number of 19 (41 percent) countries in SSA have added rotavirus vaccine to the routine infant immunization schedule. Out of these countries, only two countries introduced the Rota vaccines with no-GAVI support: Botswana and South Africa. South Africa was the first country to introduce the vaccine in August 2009 and Sudan as the first GAVI eligible country in July 2011. Other countries which have introduced rotavirus vaccine are Angola, Burkina Faso, Burundi, Cameroon, DRC, Ethiopia, The Gambia, Ghana, Madagascar, Malawi, Mali, Rwanda, Sierra Leone, Tanzania, Zambia and Zimbabwe (Table 2).

Human Papilloma virus vaccines introduction: Although uptake of human papilloma virus vaccines (HPV) in routine immunization in Africa is increasing, there are only two countries (4 percent) in Africa that have completed the roll-out of HPV into their NIP: South Africa and Rwanda. Rwanda was the first country in Africa to introduce HPV vaccine nationwide following HPV vaccine donation and support from a vaccine manufacturing company in April 201. ${ }^{35}$ GAVI began providing support for HPV vaccines in 2013and by February 2014, 20 out of 21 countries with approval of HPV vaccine support from GAVI are in SSA. GAVI started by supporting HPV demonstration projects in the first round of ten countries (Kenya, Tanzania, Malawi, Madagascar, Mozambique, Zimbabwe, Niger, Sierra Leone and Ghana) and the second round of nine countries (Senegal, The Gambia, Liberia, Cote D'Ivoire, Cameroon, Benin, Togo, Burundi and Mali) respectively in 2013 and in 2014. Further GAVI Alliance has announced that in 2014 it will support the first nationwide introduction of HPV vaccine in Rwanda for girls of alleligible ages. ${ }^{36}$ South Africa has introduced the HPV in March 2014 and became the first non-GAVI eligible country in Africa to introduce the vaccine into their NIP. ${ }^{37}$ The implementation of HPV vaccination poses challenges particularly regarding the introduction and delivery of the vaccine to preadolescent girls. ${ }^{38}$ According to USAID, 25 percent of girls in developing countries do not attend school. ${ }^{39}$ These are millions of girls potentially would be neglected by an implementation of HPV school-based vaccination in SSA countries with lower school enrollment and attendance. In a study done by Binagwaho et al. ${ }^{35}$ in addition to the existing high school enrollment rate in Rwanda, the authors show that the country has achieved high coverage of HPV with involving the community to identify girls who were absent from or not enrolled in school ${ }^{40,41}$. The achievement of the implementation of HPV vaccination in South Africa is expected as this county has one of the highest levels of school girl enrollment in Africa. ${ }^{42}$
New Vaccines Post-Introduction Evaluations (PIE): After 6 to 12 months after introduction of new vaccines, WHO recommends that countries to conduct post introduction evaluations (PIE). ${ }^{43}$ The PIE helps to assess impact of NVI on immunization programmes and learn lessons for future vaccines introductions. We found that in the last five years (2009-2014), a number of countries in SSA has conducted PIE activities. We found that during 2010-2012, there were 11 PIE reports in SSA. ${ }^{44}$ These reports have described programmatic issues related to PCV introductions.

The Childhood and adolescent immunization schedule in SSA: Referring to data of May 2014 abstracted in WHO vaccinepreventable diseases global summary, the table3and table 4display the status of immunization schedule of countries in SSA. ${ }^{45}$ The data from this WHO repository has been completed by the available information on countries that have recently added new vaccines into their NIP.

HepBand Hib immunization schedule: WHO recommends that all infants receive the HepB vaccine as soon as possible after birth preferably within 24 hours. The birth dose should be followed by 2 or 3 doses to complete the primary series. ${ }^{46}$ In most cases, 1 of the following 2 options was and still is considered appropriate:

A. a 3-dose schedule of hepatitis B vaccine, with the first dose (monovalent) being given at birth and the second and third (monovalent or combined vaccine) given at the same time as the first and third doses of DTP vaccine; or

B. 4 doses, where a monovalent birth dose is followed by 3 monovalent or combined vaccine doses, usually given with other routine infant vaccines. ${ }^{47}$ In $2013 \mathrm{WHO}$ released a position paper on Hib where it recommends that any one of the following Hib immunization schedules may be followed: 3 primary doses without a booster $(3 p+0) ; 2$ primary doses plus a booster $(2 p+$ $1)$; and 3 primary doses with a booster $(3 p+1){ }^{48}$

As most of the countries in SSA have shifted to the use of combination vaccines (Table 3 ), for the pentavalent vaccines we found that the majority of these countries have adopted the same vaccines schedule: 6,10 and 14 weeks as recommended by WHO. Equatorial Guinea has an immunization schedule of $6,10,14$ and a booster at 15 months. Pentavalent is given in other different immunization week schedule at 2, 4, 6months (Angola and Cape Verde) and 2, 3, 4months (Botswana, Burkina Faso, Congo and The Gambia). Seychelles has a different immunization schedule of pentavalent $(2,4,5$ months $)$ rather than the rest of the countries in the region. South Africa is the only country in the region that uses DTaP based pentavalent combination vaccine including IPV and Hib (DTaP-IPV/Hib) in its routine immunization program and given at $6,10,14$ week and a booster at 18 months.

As of May 2014, Mauritius was still using tetravalent (DTaPHib) vaccines and as South Africa they provide HepB monovalent vaccine in their routine immunization program at 6,10 and 14weeks. Only seven countries (Botswana, Cape Verde, Djibouti, The Gambia, Mauritania, Nigeria and Sao Tome \& Principe) in the region are giving HepB monovalent vaccine at birth. All these seven countries have also added pentavalent in their immunization program with different schedules (Table 3).

Pneumococcal immunization schedule: Among the different types of pneumococcal conjugates vaccines available on the market, there are three conjugate vaccines; 13 -valent (PCV-13), 10-valent (PCV10) and 7-valent conjugate vaccine (PCV-7). The latter is slowly being removed from the market. ${ }^{49} \mathrm{WHO}$ recommends that $\mathrm{PCV}-10$ or 
PCV-13 be introduced into immunization programs either as a three dose primary series $(3+0)$ or two doses during infancy followed by a third dose in the second-year of life $(2+1$ schedule). The choice between these two schedules should include consideration of local epidemiological factors. ${ }^{50}$ When the first SSA countries (South Africa, Rwanda and The Gambia) added the pneumococcal vaccines into NIP, they introduced PCV-7. The PCV-7 has been replaced by PCV-13 in all three countries(South Africa in April 2010, The Gambia in July 2011 and Rwanda in August 2011).We found that PCV-13 is more in use than PCV-10, in 24and 6 countries respectively (Table 4). Most of the countries (18 countries) with PCV-13 in their NIP have adopted the immunization schedule of 3 dose series of 6,10 and 14 weeks. South Africa has a 3-dose schedule at 9months. The same schedule is applicable to all six countries that have introduced PCV-10 into their immunization program. Four countries (Botswana, Burkina Faso, Congo and The Gambia) are using PCV-13 with a 3 dose series schedule of 8,12 and 16 weeks. Angola is the only country using PCV13 with a 3 dose series schedule of 2,4 and 6months.

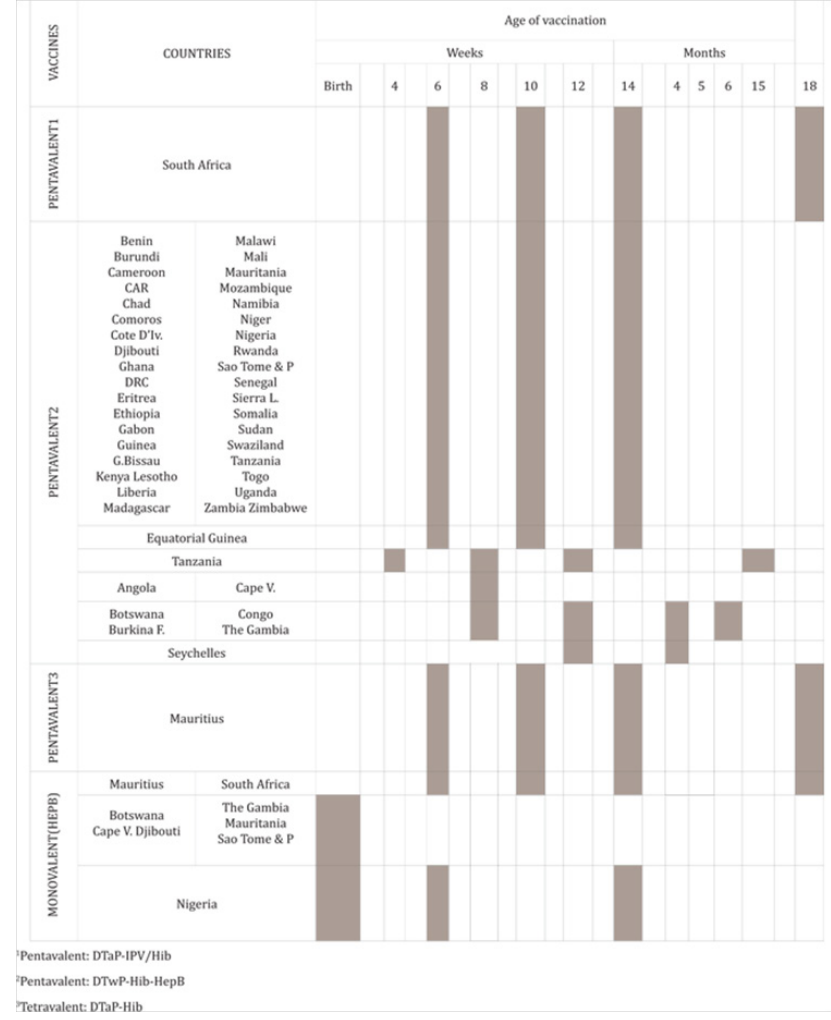

Rotavirus vaccine immunization schedule: Two oral rotavirus vaccines are marketed internationally: the monovalent (RV1) Rotarix ${ }^{\circledR}$ and the pentavalent (RV5) RotaTeq ${ }^{\circledR}$. According to WHO, RotaTeq is recommended for routine oral administration for all infants as a 3-dose schedule at the time of DTP-1/penta, DTP-2 and DTP3 contact with interval of at least 4 weeks between doses. Rotarix is recommended as a 2-dose schedule at the time of DTP-1/penta and DTP-2/penta with interval of at least 4 weeks between doses. The minimum age for the first dose of both vaccines is 6weeks. Both RV1 and RV5 are currently used in NIP of countries in SSA. By May 2014, Rotateq is being used in four countries (Burkina Faso, The Gambia, Mali and Rwanda) and 16 countries have adopted the use of Rotarix into their NIP (Table 4). The two dose series schedules that have been adopted by most of these countries are 6 and 10weeks. Botswana and Congo used the immunization schedule of 8 and 12 weeks while South Africa and Angola have $6 \& 14$ week and 8 \& 16weeks respectively.
HPV immunization schedule: As of May 2014, Rwanda and South Africa are the two only countries with HPV vaccines in their NIP in Africa. Rwanda introduced quadrivalent HPV and this vaccine is given in 3 doses for each school female adolescent from 9-15years while in South Africa, the HPV bivalent vaccine is given to girls who are 9 and 10years old.

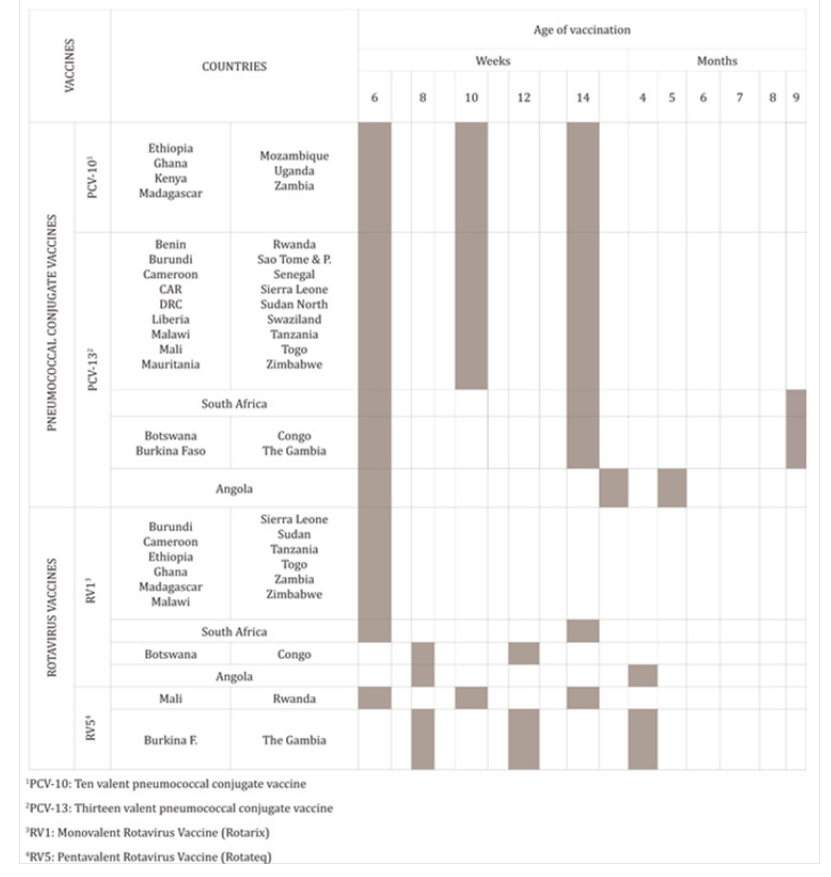

\section{Trends and Challenges for Introduction of New Vaccines}

This section will elaborate on objective number 4 , which is provide an overview on trends and challenges related to the introduction of new vaccines for routine childhood immunization in the countries of subSaharan Africa. Introducing a new vaccine in a developing country may face many several challenges. Several global health initiatives (such GAVI) have been established to overcome the challenges poor nations face while introducing new vaccines. Although the financial barrier does not today constitute the main handicap for the majority of SSA countries, so far there is still a delay within the countries in SSA during the introduction of new vaccines into their immunization program. The delay in the introduction of new vaccines has been attributed to multiple factors. A study of Hajjeh et al., ${ }^{51}$ identified these factors which can be summarized under three major categories:

i. Lack of recognition of the value of a vaccine,

ii. Factors related to decision making process and

iii. Policy considerations. ${ }^{51}$

The paragraphs below will further elaborate on these three categories.

\section{Value of A Vaccine and Time of Introduction}

With regards to the value of a vaccine, the authors highlighted the importance of the availability of local data on disease burden, costeffectiveness and documenting on efficacy of the vaccine in various parts of the world, and not only in developed countries. We found that for the both HepB and HibCV vaccine there has been a considerable delay in reaching the first child in the last country of SSA. HepB got 
its first licensure in U.S. in 1986 and received WHO prequalification as well as its first WHO recommendation for routine use one year and five years later respectively. The Gambia was the first country in the SSA to introduce this vaccine. The first studies on vaccine efficacy of HepB in SSA were conducted in 1993 by Fortuin et al., ${ }^{52}$ which was supported by a study done by Viviani et al., ${ }^{53}$ in $1999 .{ }^{52,53}$ The same year as the efficacy study was conducted, Hall et al., ${ }^{54}$ carried out a vaccine cost effectiveness study. ${ }^{54}$ From the time of the first vaccine licensure in a developed country (USA in 1986), ${ }^{55}$ it took approximately 4years for the vaccine to be introduced in The Gambia. We found out that, it has required 27years from the date of licensure to reach the last country in the region of SSA.

Following the first licensure of HibCV in 1986 in Canada, ${ }^{56}$ it took a period of more than 10years to see WHO prequalifying and recommending the vaccine. The first published results on vaccine efficacy and cost effectiveness of HibCV were done in The Gambia in 1997 by Mulholland et al., ${ }^{57}$ and in South Africa in 1995 by Hussey et al. ${ }^{58}$ respectively. Therefore, The Gambia introduced also the HibCV as the first country in SSA eight years after the first developed country (Iceland in 1989) had added this vaccine into their NIP. ${ }^{31}$ From the year of licensure of the vaccine, it took a period of 27years (quite similar to HepB) until the two last countries (Somalia and Equatorial Guinea) in the region of SSA had added this vaccines into their NIP.

Despite the fact that other new vaccines such as RotaV, PCV and HPV have not yet been added into the immunization program of all SSA countries, the following section will focus on the time that has been required for the first country to introduce these new vaccines into its immunization. The two available Rotavirus vaccines; monovalent (RV1) and pentavalent (RV5) got their first licensure in Mexico (RV1) and U.S. (RV5) in 2004 and 2006 respectively, ${ }^{59}$ and they have both been recommended for routine use by WHO in 2009. Thus, it took 2-3years for WHO to prequalify these two vaccines. We found that the first published result on vaccine cost effectiveness on RV1 was conducted in Malawi in 2010 by Berry et al., ${ }^{60} \mathrm{RV} 1$ got introduced for the first time into the NIP of South Africa one year before availability of data on vaccine efficacy in South Africa and Malawi. This study on efficacy was conducted in those two countries by Madhi et al., ${ }^{61}$ The data of RV5 from a study in SSA on vaccine efficacy and cost effectiveness were available as of 2010 and 2012 respectively. In 2010,Armah and colleagues conducted the first study on vaccine efficacy in SSA in three countries Ghana, Kenya and Mali while the first available data on cost effectiveness of RV5 were done by Abbott and colleagues two years after. ${ }^{62,63}$ Rwanda became the first country in the region of SSA to introduce RV5 after 6years from the first licensure.

The first clinical trial data on vaccine efficacy on published on PCV vaccines in SSA was done withPCV-9 in 2003 and 2005, respectively in South Africa by Klugman et al., ${ }^{64}$ and in The Gambia by Cutt et al., ${ }^{65}$ The PCV-9 has not been introduced; this vaccine was abandoned, while PCV-7 was licensed. In 2010 in The Gambia, Kim et al., ${ }^{66}$ conducted the first SSA country-specific cost-effectiveness analysis of the vaccine. ${ }^{66}$ The Gambia and Rwanda became the two first countries in the region to add PCV-7 to their NIP nine years after the first licensure in U.S. and as first developing country to introduce the vaccine.$^{67}$ Kenya became the first SSA country to introduce PCV10 into their immunization program in February 2011, one year after the WHO PQ and two years after the first licensure of the vaccines in Canada (December 2008). PCV-13 was approved first in Chile in July 2009 and Mali introduced PCV-13 as first SSA countries in March 2011. ${ }^{31,68,69}$ Today, PCV-10 and PCV-13 are much more widely used than PCV-7 in the NIPs of SSA countries.
With only two countries that have introduced HPV vaccine in their immunization programs so far, Rwanda introduced the quadrivalent (HPV-4) in 2011, this was two years after WHO PQ and five years after the first vaccines were licensed and introduced in USA. ${ }^{35,70}$ South Africa introduced the bivalent (HPV-2) in 2014, that is 8years after the vaccine got its first licensure in Australia in 2006. ${ }^{71}$ Even though the HPV vaccines are currently being introduced in SSA, to-date there is no clinical data published on the vaccine efficacy and effectiveness in any of the countries of SSA on HPV-2 and HPV-4. ${ }^{72}$

\section{Decision making process and policy consideration}

Understanding better the decision-making process during the introduction of new vaccines is critical to establish why vaccines are adopted or not. It also contributes to building a sustainable demand for these vaccines in a country. The purpose of this section is to map and analyze the formal decision-making process in relation to the introduction of new vaccines within the context of countries of sub-Sahara Africa. In a study done by Mantel and Wang, the authors highlighted a multitude of factors influencing developing countries (including most of the SSA countries) decisions to introduce new vaccines, and the process for making these decisions. ${ }^{73}$ These factors are

\section{i. Sources of information and the decision-making process,}

ii. Quality of data for decision making,

iii. Vaccine characteristics,

iv. Immunization financing and

v. Immunization delivery systems.

The following paragraph will elaborate these five factors.

\section{Sources of information and the decision-making process}

One of the key and undoubted sources of information for decision making on NVI in countries in SSA is the World Health Organization (WHO) global recommendations and policy guidelines, (i.e. the vaccine position papers). Another influential factor for decision making on NVI is the availability of donor support. To date the majority of new vaccines introduced into NIP of countries of SSA have been done mainly through financial support from GAVI. The role of donors in vaccine introduction will be discussed separately in another section.

Data for making decisions and vaccine characteristics: In the review of Mantel and Wang, the authors found that the quality of local or regional data on the burden of VDPs is critical for the decisionmaking process in NVI. In a number of countries, vaccines would not have been adopted if local disease burden data had not been available. ${ }^{73}$ The authors gave an example of Sudan where the decision to prioritize rotavirus vaccine over other potential new vaccines was based on the availability of local data demonstrating a high proportion of rotavirus disease in children hospitalized with diarrhea.

The local data are often collected in sentinel hospitals, which form part of global surveillance networks, coordinated by WHO. In a study done by Gordon et al. ${ }^{74}$ vaccine characteristics appear to have limited influence on decision-making processes in low-income countries such as the majorities of countries in SSA. ${ }^{74}$ In the last decades, WHO and different partners have attempted to enable countries of SSA to define and further develop their own regulatory policies. Based on the available data from WHO VPD: monitoring system global 
summary and Observatory of the National Immunization Technical Advisory (NITAG) Resource Center by the 2011, out of 48 countries in SSA, only 13 (27 percent) have established a functional stringent national regulatory agency. ${ }^{75,76}$ These include Sierra Leone, Liberia, Mauritania, Cote D'Ivoire, Nigeria, CAR, Ethiopia, Angola, Zambia, Mozambique, Zimbabwe, Madagascar and South Africa. Among these, 60 percent meet the required standards. ${ }^{77}$ WHO defined NITAGs as a technical resource which provide guidance to national policymakers and programme managers to enable them to make evidencebased immunization-related policy and programme decisions. NITAG should have a legislative or administrative basis for the advisory group, a team of experts such as pediatricians, public health professionals, infectious disease experts, epidemiologist, immunologist and other experts. In addition to this, this advisory group should have formal written Terms of Reference. ${ }^{76,77}$

Immunization financing and sustainability: Burchett et al., ${ }^{78}$ conducted a study in which they highlight the availability of external vaccine support, such as through the GAVI Alliance, as the major driver for adopting decisions about introducing new vaccines into the NIP of low incomes countries. ${ }^{78}$ In line with this, financial sustainability has been considered as the hindrance in many countries in the region of SSA, specifically those with ongoing external support.

In the study conducted by Machingaidze et al., ${ }^{26}$ in year 2005, 49 percent (26/53) of African countries reported that at least half of the costs of their routine vaccines were funded by their own governments. ${ }^{26}$ However, of those that established line items in their national budgets for routine vaccines, over a third of them did not fund them, and those that had drawn financial plans did not utilize them to the expected degree. Only 10 percent $(5 / 48)$ of SSA countries reported on routine vaccines funded solely by government (GoV) in 2012. These countries include Angola, Gabon, Mauritius, Namibia and South Africa. Among the SSA countries with lower percentage (less than 5) of routine EPI vaccines financed by GoV, we found Guinea, Djibouti and Somalia $(0$ percent), Sudan ( 1 percent), CAR ( 2 percent), Eritrea ( 3 percent) and Madagascar (4 percent). Overall, we found only six countries in SSA that have equal or greater than 50 percent of their GoV budget to finance the routine vaccines. These countries are Seychelles (60 percent) and the five countries mentioned above.

GAVI has long been concerned with the sustainability of immunization programmes and their benefits, an issue that is coming to the fore as countries cross the income eligibility threshold and start to graduate from GAVI assistance. GAVI's graduation process is designed to ramp up domestic co-financing of vaccines while GAVI financing slowly phases out over several years, so that once GAVI support ends, the country will be able to fully fund these vaccines (and associated costs) in a fiscally sustainable way. In a study conducted by Saxenian et al., ${ }^{79}$ the authors found that out of sixteen countries that are scheduled to 'graduate' from GAVI assistance by 2018, only two countries Angola and The Republic of Congo are from Africa. ${ }^{79}$ For the Republic of Congo, GAVI is supporting new vaccines such as pentavalent, pneumococcal and yellow fever and has approved the introduction of rotavirus vaccine. To pay for these four vaccines, Congo's co-financing will need to increase from about US\$90 000 in 2011 to US\$ 3.0million in 2015 and US\$ 4.0million in 2016. This is estimated to account for 0.8 percent of the Ministry of Health's budget in 2015 and 1.0 percent in 2016. However, the transition planning team expressed its concerns about the effectiveness of government to ensure that the required funds are budgeted, approved and channeled to the health ministry. Congo's default in 2012 on its GAVI cofinancing payments highlighted the magnitude of the problem.
Immunization delivery systems: Burchett and colleagues found that the capacity and quality of the vaccine delivery system is another important area to consider when making decisions on new vaccines introduction. ${ }^{78}$ Before introduction of new vaccines, prior preparations based to the analysis of the capacity of the cold chain and logistics systems including stock, transport and waste management are extremely important.

As for any other new health interventions, decisions to introduce new vaccines should take into account the aspect of communication and social mobilization strategies. These include crisis communication plan in case of adverse events, as key to ensuring public acceptance. Burchett and colleagues highlighted that health workforce is one of neglected area of concern when adopting new vaccines. ${ }^{78}$ Decisions to introduce a new vaccine may result in an increase in workload and complexity of administering vaccines, and an increase in requirements for the number, distribution and capacity of health care workers. Mantel and Wang mentioned other reasons can push a nation to decide introducing new vaccines including the readiness of local manufacturers to produce a particular vaccine and the political pressures towards reaching MDGs. ${ }^{73}$ The acceleration of the introduction of new vaccines such as Pneumococcal and Rotavirus vaccines in the region of SSA is among the priority to reach MDG$4 .{ }^{80} \mathrm{We}$ found that a partnership between a nation and manufacturing company can influence can to introduce the vaccines. The first introduction of HPV in Africa resulted from a donation between Merck and the government of Rwanda. In this partnership, Merck has agreed to donate approximately 2 million doses of Gardasil over a period of three years. ${ }^{35}$

Policy considerations for NVI: The policy considerations are the third categories that the following sub-section will elaborate as factors causing the delay of vaccines introduction into immunization programs. In the study carried out by Hajjeh $\mathrm{R},{ }^{51}$ the author found that policy considerations play an important role in supporting vaccine decisions as well; in particular, the absence of clear global recommendations is a strong negative factor. ${ }^{51}$ For example, in the case of Hib vaccine, the initial WHO position paper did not provide a strong, supportive recommendation for vaccine use but was rather weak and permissive, and as a result, did not generate significant demand for the vaccine. ${ }^{81}$ The revision of this position paper came two year later in 2006 into a steady recommendation calling for universal vaccine introduction in all countries sent a strong and clear message to countries about the value of Hib vaccines. ${ }^{82}$ Similarly, the absence of clear financing policies and commitments from donors can negatively impact vaccine decisions, as countries become reluctant to commit their limited resources (For instance the implementation of birth dose HepB vaccine, as described previously). Besides these global factors, we found that regional factors can also play an important role. In the study carried out by Shearer and colleagues, the decision of neighboring countries to introduce a vaccine is independently associated with accelerated decision-making in a country. ${ }^{83}$ Looking at the country level, there is often a lack of awareness about the value of vaccines, their efficacy and safety, potential contribution to national health priorities and mortality reduction, and a poor understanding of local disease burden issues. ${ }^{51}$

\section{Proactive planning and funding opportunities}

A major effort to introduce new vaccines into the nations of the region of SSA was initiated in recent years with the help of the GAVI alliance. Since its founding in 2000, the GAVI alliance provides cash support to the majority of governments in the countries of SSA to strengthen the health systems that are responsible for delivering new 
vaccines as well as for traditional vaccines. As of May 2014, GAVI has conducted and approved 3 phases (phase 1 of 2000-2005, Phase 2 of 2006-2010 and phase 3 which is ongoing 2011-2015). Under each of the GAVI phases, the alliance provides financial support to its eligible countries, which usually includes new vaccines, safe injection supplies, and Immunization Services Support (ISS) funding. The first GAVI supported vaccines introduced in countries of SSA have been the Hib and HepB vaccines. The introduction of these two vaccines occurred during the first phase of GAVI's operation sand demonstrated considerable variability to a substantial number of countries of SSA.

In the study conducted by Glatman-Freedman A et al. ${ }^{84}$ the authors found that, not only the availability of funding were behind the success of the introduction of the new vaccines into the immunization program of countries in SSA. ${ }^{84}$ The authors identified 3 distinct groups of countries from SSA based on the status of NVI or use during the first phase of GAVI's operation (2000-2005) (Figure 1). The first group (group I) of countries in which both Hib and Hepatitis B vaccine were introduced includes Benin, Burkina Faso, Burundi, The Gambia, Ghana, Kenya, Malawi, Mali, Rwanda, Senegal, Uganda and Zambia. Note that The Gambia started HepB and Hib vaccination program on its own (respectively in 1990 and 1997) and was approved for GAVI's support for these vaccines in September $2000 .^{85}$ The second group of countries (group II) was countries in which only hepatitis B vaccine (not Hib vaccine) was introduced. This group included Cameroon, Comoros, Cote D'Ivoire, Eritrea, Lesotho, Madagascar, Mauritania, Mozambique, Nigeria, Tanzania and Zimbabwe. The third group (group III) comprises countries in which neither Hib nor Hepatitis B vaccine were introduced and includes Angola, Chad, CAR, Congo, Congo DR, Ethiopia, Guinea, Guinea-Bissau, Liberia, Niger, Sierra Leone and Togo).

Among the main determinants evaluated behind the success of introduction of new vaccines into NIP of the these 3 group of countries, two elements play an important role

i. Financial indicators related to healthcare and

ii. Country-level governance indicators. There were no statistical differences with other evaluated indicators such as healthcare indicators such as life expectancy and number of available of doctors and nurses.

Financial indicators related to healthcare: The financial indicators related to healthcare evaluated and identified were: Total Healthcare Expenditure per Capita (THECAP), Government Healthcare Expenditure per Capita (GHECAP) and Total Healthcare Expenditure as percent of the GDP(HEGDP). For GHECAP, statistically significant differences were found between group I and III for the years 2002, 2003 ( $\mathrm{p}$ value $<0.05$ ), 2004 and 2005 ( $\mathrm{p}$ value $<0.01$ ) (Figure 1). For HEGDP, the differences between groups I and III reached statistical significance in the years 2004 and 2005 ( $\mathrm{p}$ value $<0.05$ ) (Figure 1). For THECAP, the pattern of expenditure was similar to that of GHECAP, but no statistically significant differences were demonstrated between the groups (Figure 1)

Country-level Governance Indicators: Country-level Governance Indicators scores for each country were obtained from the World Bank database. These included: Political Stability, Government Effectiveness, Rule of Law, Regulatory Quality, Control of Corruption, and Voice and Accountability. Gordon and colleagues conducted a study to explore the interest and opportunities for the proactive planning of new vaccines introduction in low- and lower-middleincome countries. ${ }^{74}$ The study has looked at some of the countries of SSA which introduced multiple new vaccines into their NIP.
These countries include Ghana (rotavirus, pneumococcal), Tanzania (rotavirus, pneumococcal), Uganda (rotavirus, pneumococcal), Zambia (rotavirus, pneumococcal) and South Africa (rotavirus, pneumococcal and pentavalent vaccines). The authors found several characteristics for countries that are proactively planning for the introduction of multiple new vaccines, including an

I. Increased attention to systematically scaling up the cold chain and vaccine logistics systems,

II. Positive modification to reporting procedures, and the

III. Expansion of training curricula. Other benefits noted were

IV. Economies of scale are most likely to be realized when the new vaccine easily integrates into the routine schedule for delivering an existing vaccine. For instance, because of the pre-existing human and logistical resource limitations within South Africa's immunization programme, exacerbated by efforts to integrate immunization with other health services, the introduction process was argued to be a strain on some of the immunization programme's already overstretched systems.
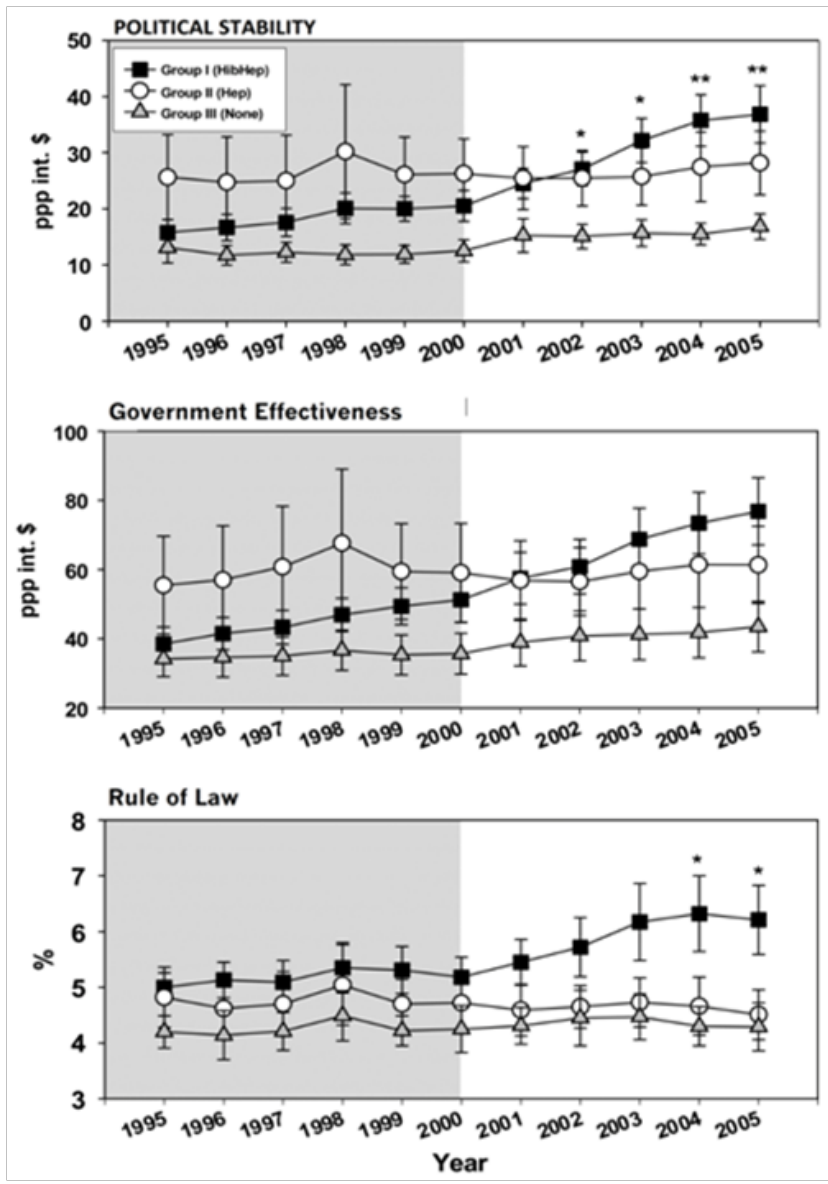

Figure I Financial healthcare indicators during the pre-GAVI and the first phase of GAVl's funding in group of countries from SSA.

\section{Political opportunity for Africa leaders}

Political commitments from African governments are among the key factors that both maintaining current achievements and making additional progress for EPI in Africa. ${ }^{26}$ In the most of developing countries including countries of SSA, Health Ministers or their representatives have committed themselves to introduce new vaccines into national immunization programs. However, in the last couple of 
years, the introduction of new vaccines in Africa has been seen as an important event in which it is required the presence of more senior political leaders.

The table 5 displays the available information on the involvement of Office of Head of States or First Ladies while new vaccines were being launched in SSA during the last five years. During this time the event to launch and introduce new vaccines into the NIP in SSA has been seen as an important event which involved the presence of president or vice president. The national launch of pneumococcal vaccines in Sierra Leone, Uganda, Kenya has been presided by the head of states. ${ }^{86-88}$ The Vice president from Burundi, Malawi and The Gambia took part in the launch of other different new vaccines into their respective countries. ${ }^{89-91}$ In most of the countries in SSA, the involvement of First Ladies in launching and introduction of new vaccines is increasing. We found out, First Lady from Burkina Faso, Cameroon, DRC, Ghana, Rwanda, South Africa, Tanzania, Uganda and Zambia attended the ceremonies to launch and introduce of new vaccines at national level. ${ }^{92-101}$

\section{Discussion}

\section{Status vaccines introduction and immunization schedules}

There is now sufficient data and information to show that there have been major improvements in introducing new vaccines into the NIP of countries of SSA during the last 40years. At the date of the $40^{\text {th }}$ year's anniversary of the establishment of the EPI, HepB and HibCV vaccines have been completely added into all immunization program of SSA Africa and the introduction of PCV and RotaV is ongoing. So far it has reached 66 and 41 percent for PCV and RotaV respectively. Our results is comparable to the study done by ChaukeMoagi BE \& Mumba $\mathrm{M}^{102}$ in which 2years ago, in SSA region there were no completion of introduction of any of these vaccines. ${ }^{102}$ All this, thanks to commitment of governments of SSA countries and their partners.

We found that geographical location and socio-cultural factors might play an influential role to introduce new vaccines in SSA. Due to the dynamicity and competition among countries in the same sub-region, this might explain the reasons that countries introduce the vaccines in the same period. Sociocultural factors influence the decision for vaccines introduction. These factors need to take in consideration the opinions of parents, children and community. The important of these factors have been seen in a study done by Bingham et al., ${ }^{103}$ on the assessing the introduction of HPV. ${ }^{103}$ Two examples from Uganda in this study can illustrate the importance of the socioculture factors for NVI:

Example 1: A mother in Uganda explained, "Parents may refuse to let children go to a film, but if the children are interested they will surely escape through the windows and go out. Similarly, if children choose to be vaccinated at school, while their parents are busy attending to their farms, they will get home and refuse to entertain questions from their parents about immunization".

Example 2: A religious leader in Uganda also expressed the following point: "As leaders we will need to know where the vaccine is coming from, whether the vaccine has side effects or not, whether it is not just being tried on people, where it has worked, and all the other details pertaining to it like has it been approved by the World Health Organization."

In the sub-region of SSA Central Africa, the majority of countries have added HepB and Hib into their NIP on period of 2-3years. The same is seen in other sub-regions. Most countries of the Eastern Africa region as well as a half of the countries of Southern Africa have introducing HepB in the beginning of 2000. The majority of the countries of these two sub-regions introduced Hib vaccine in form of combination later in 2000s. In spite the fact that some countries in SSA have shown their strong engagement in adding the new vaccines into their NIP there is 20years between the first country and the last country in SSA to complete the roll out of the new vaccines into their NIP. At country level, three countries The Gambia, Rwanda and South Africa are heading in SSA to introduce these new vaccines. To-date, Rwanda is the only country in SSA that has completed the introduction of all the twelve routine childhood antigens recommended by WHO as use of all. The achievement of introducing the routine childhood vaccine in Rwanda as well as South Africa demonstrates the strong commitment and leadership towards the importance of vaccines in fight against child mortality. Rwanda is cited as having one of the strongest immunization programs in Africa and demonstrates a good example of the role of partnership between the SSAGoV and donors in introducing new vaccines. ${ }^{35,104}$ Besides the acceleration of NVI, such countries in SSA like South Africa, they have shown strong engagement for securing funds to sustain its immunization program. ${ }^{79}$

New Vaccines PIE in SSA. Our findings show that the majority of SSA countries have introduced the PCV in the period of 2010-2012 (Table 2). However, the 11 PIE reports found doesn't correspond to the total number of countries introduced the PCV in the above period. Therefore, the PIE activities in SSA are not carried by many countries following the NVI. In countries where PIE activities have been conducted, the introduction of new vaccines created some problematic issues. ${ }^{44}$ First of all, the NVI might accompany in shortages of new vaccines for the target age population. At the first day of vaccination, children outside the target infant age group also were also brought. Secondly, health worker were mainly focusing on new vaccines introduced and do not give time to educate the caretakers about other prevention and treatment options (caseof pneumonia for PCV introduction). Thirdly, concerns related to the capacity of the immunization systems. These include needs for

i. New training methods to address the complexity of messages relating to use of new vaccines,

\section{ii. Improvement of supportive supervision, and}

iii. Further enhancement of injection safety, injection waste management, and monitoring of adverse events after immunization (AEFI).

\section{NVI in countries with high burden of diseases}

At the date of the anniversary of EPI, a group of countries with high of burden of diseases have not yet added their vaccines into their NIP. A country like Nigeria has not yet introduced neither PCV nor rotavirus. In the recent and local study conducted at the South East of Nigeria by Tagbo et al., ${ }^{105}$ the rotavirus related disease is causing over 160,000 under-five deaths yearly and the place Enugu has the highest incidence rate in Africa. ${ }^{105}$ The authors found that out of 615 diarrhea stool samples collected, 56 percent were positive for human rotavirus. Among these positive samples, 96 percent were children under age of 2 while 77 percent were less than one year of age. The authors highlighted the urgent need for introduction of rotavirus vaccine into Nigerian immunization program. Other countries such as DRC and Uganda have introduced PCV and not yet rotavirus. Over the past 2-3years, considerable efforts have been made in Angola and Ethiopia as these two countries have completed to roll out the PCV and rotavirus vaccines. Considering that in the region of SSA millions 
of children's lives a year could be saved by a simple and cost-effective intervention Immunization, Africa leaders should take action; these life-saving vaccines should be their priority in the new few years.

\section{Standard routine immunization schedule}

The findings of the review have noticed that the majority of countries inSSA have adopted a national standard routine immunization schedule for infants at 6,10 , and 14 weeks of life. This immunization schedule is the one recommended by WHO and constitutes the basis of traditional vaccines such as Bacille Calmette-Guérin (BCG) and oral polio vaccine (OPV) given at birth, followed by DTP and OPV, Hib and HepB at 6 weeks, 10weeks and 14weeks. Also with the PCV and RotaV most of the countries of SSA have adopted this standard schedule. In comparison to Europe the countries in SSA stand better when it comes to having a uniform immunization schedule. In the study done by Wiese-Posselt et al., ${ }^{106}$ looking at the potential benefits of harmonizing immunization schedules of countries, ${ }^{106}$ the authors found that a uniform immunization schedule in a region may facilitate mobility of citizens, improve data collection and increase vaccination coverage either quantitatively or qualitatively. On the other hand the some factors such as values and goals, epidemiological situation, health care delivery system and logistics of vaccine delivery and economic situation may differ substantially between countries and thus support different immunization schedules, This might explain the initiative launched by the European Commission (EC) through a Workshop of Vaccination Strategy held in Luxembourg in 2008 and again strengthened in the European Science Advisory Network for Health (EuSANH)workshop in Malta, 2012. ${ }^{107,108}$ The 2012 EuSANH workshop emphasize that the harmonization of vaccination schedules in Europe is not a priority, but the reasons behind the differences should be explained in an understandable and coherent way to the public. The difference among the immunization can lead to the public questioning: for instance why the same vaccine product is utilized in different ways (dosage and timing) in different countries where the epidemiological situation is fairly similar and it won't be an easy job for health care worker to explain reasons. The distrust might rise not only amongst people deciding on their own or their children's vaccinations, but also amongst health care workers, journalists and politicians alike

Birth dose of Hepatitis B vaccine: As of May 2014, HepB was administered in all countries in the region of SSA. Besides this completion, according to WHO, in 2009 more than 4million infants in the African Region did not receive three doses of the HepB vaccine. ${ }^{109}$ Currently only seven countries in the SSA Region (Botswana, Cape Verde, The Gambia, Djibouti, Mauritania, Sao Tome Principles and Nigeria) are reporting administering a birth dose, and the timeliness of these doses is unknown. ${ }^{109}$ There is no publication from these four countries comparing the effectiveness of a birth dose to one given at a later age. Recent studies have shown that maternal-fetal transmission (MFT) plays an important role as route of transmission in contrast to the old studies finding that MFT play a relatively minor role. ${ }^{110-112}$ Today, it is very clear and documented that African mothers can also infect their newborn infants. A study done by Kramvis et al., ${ }^{113}$ has look at whether it may be possible to implement a birth dose of hepatitis B vaccine for home deliveries in Africa. ${ }^{113}$ It is relatively easy to immunize babies born in health facilities but if it is required to reach home deliveries within 24 hours of birth, this pose severe challenges to many resource-poor countries in SSA.

There are significant opportunities to improve the timely delivery of HepB vaccine birth dose in existing maternal and child health programmes where health workers are trained to provide home delivery care. Therefore, for a number of sound reasons related to delivery of health care for the new mother and infant, it seems likely that an increasing numbers of home births will be attended in Africa by trained health staff in the future. In time, this will open the way for the administration of the vaccine outside the cold chain using technologies such as the disposable needle and pouch (UnijectTM). Financial barriers might be another obstacle in implementing the HepB birth dose into the NIP of countries of SSA. Medecins Sans Frontieres (MSF) expressed its concern to GAVI about the slow implementation of the HepB birth dose vaccine immediately after birth as recommended by WHO. ${ }^{114}$ In responding to MSF, GAVI as the main financial support of new vaccines in SSA, has decided not to offer financial support for the birth dose to any country. ${ }^{115}$ According to GAVI, there are significant challenges other than the price of the vaccine for the implementation of the practice. Many of the births in GAVI-eligible countries also occur outside health care facilities, which would make it difficult to administer the vaccine in the twentyfour hour window after birth. Our review findings show that the implementation of birth dose of HepB vaccine in SSA is still a debate. HBV transmission from mother to child in the early perinatal period exists and remains a significant contributor to the burden of HBVrelated disease in SSA, therefore our review support the conclusions from a study done by Howell et al., ${ }^{116}$ namely the prevention strategies tackled on maternal/child transmission (including HBV birth-dose vaccination) and early horizontal transmission of HBV should be implemented in SSA. ${ }^{116}$

\section{Decisions and timeliness of vaccines introduction}

The decision-makers should reflect the potential effect of the introduction on both the programme as well on the overall health system while deciding the introduction of a vaccine into the NIP. In the case, there are serious deficiencies in the immunization programme, adding the new vaccine may originate supplementary burdens and thus deteriorate the performance of the program. If there is a failure of immunization program in reaching a large number of populations in need, the benefit of the new vaccine will limit to those who need it most. In such cases, delaying the NVI while addressing programme weakness may be the best choice. It is obviously that all NIPs in SSA do not have the same capacity of handling the problems related to their immunization program. However, considering the speed and scale up of NVI in SSA, decision-makers should take to account, the best option to build the capacity of their program. Before the introduction of the new vaccines, decision-makers must also decide whether the NIP and health systems have capacity of handling, storing and administering the vaccine adequately, considering its specific characteristics (dosing, schedule, heat stability, freeze tolerance) and cold storage space requirements. Looking beyond of the intrinsic aspects of immunization programme, the shortage of health worker in Africa represents in general a major barrier to scaling up new intervention at the community level. ${ }^{117,118}$ However, if the current NIP workforce is insufficient and has inadequately trained or poorly motivated personnel, introducing a new vaccine to the programme may consequently resulting in more stress on these workers, poor coverage of the new vaccine as well as those already in the programme. It is very curious to conduct a review of the immunization programme in order to detect weak areas before a vaccine is introduced. When weaknesses are initially identified, they can be tackled and explicitly strengthened in the process of introducing the new vaccine.

A number of important elements need to be put in place for a country in SSA to be ready before introduction of the new vaccines. These important elements take account of the communication and advocacy strategies, the increase and training health care workforce 
and the revision of informational materials (these include registers, immunization cards and forms for monitoring and evaluation of immunization services). The training for health care staff is essential because they are responsible for handling and administering new vaccines and they are a major source of information for parents and others in the general public. The development and introduction of new vaccines is a costly and time consuming process. Unfortunately, those most in need, individuals in developing countries, are the last to receive these powerful disease preventing products. From the time a vaccine is licensed in a developed country to the time most of the poor in developing countries have access to the vaccine 20-30years may have passed. ${ }^{119}$ In the region of SSA, the two vaccines HepB and HibCV that have been rolled out has required a considerable delay to reach the people targeted for vaccination. Our findings show that it took 4years from the vaccine licensure to see the first country introducing and 27 years to reach the last country to introduce this vaccine in the region. This delay is unacceptable and could therefore be considered as lessons to be learnt while introducing the current new vaccines (PCV, RotaV and HPV). These lessons could also be considered as cornerstones for other future potential vaccines of public health importance which are in the advanced stages of clinical development. These vaccines might be available on the market in the next few years and include malaria, dengue, typhoid conjugate vaccines and new-generation tuberculosis vaccines.

\section{Partnership opportunities and African leadership}

The GAVI Alliance's initiative to introduce new vaccines into developing countries is of utmost importance for the health of children worldwide. The Alliance's role in providing the financial resources for this purpose is crucial, especially given the high costs of such vaccines. Examining the first phase of GAVI's funding, we found that GAVI-eligible AFRO countries differed with respect to the introduction of new vaccines into their immunization programs. While some countries introduced both the Hib and HepB vaccines (Group I), other countries introduced only the HepB vaccine (Group II). A third group of countries did not introduce any of the vaccines. The stable pattern of governance scores for the 3 country groups before and during GAVI's first phase of funding, and the association of higher governance scores with the introduction of both HepB and Hib vaccines (country group I), solidly support the strength of governance as an important factor in the ability of countries to support healthcare initiatives, including the introduction of new vaccines. These patterns strongly indicate that governance is a stronger predictor for the introduction of new vaccines.

Overall, our findings indicate that country-level governance is the most important factor in determining the ability of poor African nations to introduce new vaccines. Good governance offers an obvious advantage for a country's ability to move forward with new healthcare initiatives like the introduction of a new vaccine. New efforts require the commitment and attention of leadership at the top governmental levels who must obtain and commit funds over multiple years. In nations that are struggling financially, political stability and good governance are often important factors in attracting foreign aid. In this regard, good country-level governance was previously shown to have a considerable impact on investments in developing countries made by for-profit healthcare service providers and large multinational pharmaceutical and biotechnology corporations. ${ }^{120}$ In addition to funding, referring to the study done by Duclos et al., ${ }^{121}$ the authors emphasize that the introduction of new vaccines requires adequate infrastructure elements such as sufficient cold chain capacity, ability to reach remote locations, safe disposal of needles and syringes, as well as sufficient numbers of adequately trained personnel. ${ }^{121}$ Lack or scarcity of these elements constitutes significant barriers to the adoption of new vaccines. Country-level governance may have a crucial effect on both the initiation and mobilization of these important elements of vaccine programs.

Vaccination is a fundamental human right that must be protected through the political process. ${ }^{122}$ Political leaders recognize that childhood immunization is a cost-saving endeavor that returns much more to society than it costs. ${ }^{123}$ However, immunization sometimes suffers low political commitment due to many competing health priorities. During the acceleration phase of introduction of new vaccines into the NIP of SSA countries, the political commitment in SSA is often manifested as support from the highest levels (e.g., President, Prime Minister, and Minister of Health). In the recent years, strong involvement of First Ladies in launching the new vaccines as well as immunization campaign in many SSA countries has been noted. Although this positive development cannot be underplayed, it is also important that a commitment for immunization is present at the district or local government level. In addition to this participation of leaders, the successful of introduction of new vaccines cannot reach without the required of the public engagement. In the study review of Paterson and colleagues, the public comprise many various stakeholders. These include individuals, parents, policy-makers, researchers and clinicians and immunization programme managers. ${ }^{124}$ The polio boycott in the northern of Nigeria in 2003 was the good example in which the local religious leaders have first negatively and then positively influence parents towards the polio vaccine. This example of Nigeria shows the importance of social networking in introduction of new vaccines. The immunization program managers can profit from engaging local religious leaders in discussions about the needs of their community and how best to meet them. ${ }^{125}$

Even with continued support from donors, political will as well as financial planning and commitment from African governments will be key factors for successful introduction and sustainability of new vaccines in EPI schedules in Africa. ${ }^{26}$ The introduction of a vaccine into the NIP may also present opportunities to improve the immunization and health systems. For example, the training of health workers for the new vaccine presents opportunities to refresh their skills and knowledge in immunization and other related health services. With the existing support of partners, African leaders should the lead to create and strengthen the NITAGs in order to make evidence-based decisions, improve planning and upgrading of the cold and logistic system for NVI.

\section{A look beyond $40 y e a r s$ anniversary of EPI in SSA}

Immunization is considered the most and simple cost-effective savings a country can make to secure a healthy workforce, safeguard its population against diseases, outbreaks and push its economy into the next stage of development. In comparison to the progress so far made by countries since the inception of EPI four decades ago, there have been significant improvements in the performance of the EPISSA region. On the occasion of the 40years anniversary of EPI, Margret Chan, the Director-General of WHO published an article statement. ${ }^{126}$

The author acknowledged the role of EPI in encouraging new model of international cooperation, new source of funding and stimulating new technologies. The creation of the GAVI Alliance facilitates SSA countries to introduce new vaccines into their NIP by reducing the vaccine cost and make them affordable. However, national immunization budgets are still currently insufficient to sustain the programs and incorporate new and costlier vaccines. Most of the countries in SSA remain heavily dependent on foreign financial support. Africa has the potential to fund its own development 
agendas through several untapped resources. WHO, African Union or countries under their sub-regional community should learnt from the long experience and success story from Latin American in which the PAHO has established the Revolving Fund for Vaccine Procurement. Since its inception in 1977, the Fund has helped to vaccinate millions of children and save their lives. The presence of this fund, can explain the reason that he Region of the Americas was the first of the six WHO regions to eliminate polio. At the same time, the Fund has facilitated the rapid and equitable introduction of new vaccines. ${ }^{127}$ It is time to minimize different narrative declarations and turn around to new strategic to overcome dependency on foreign financial support on immunization which should be encouraged and supported by African leaders. The latter should prioritize sustainable immunization programmes as part of continued investment in the healthy futures of children and families in their countries.

\section{Conclusion}

As one of the most successful public health interventions, immunization plays a major role and significant stride towards reducing child deaths. The World Health Organization (WHO) established the Expanded Programme on Immunization (EPI) in 1974 with the overall purpose to make vaccines accessible to infants, children and mothers worldwide. Six vaccine preventable diseases (VPDs) were initially included in the EPI. These included tuberculosis, poliomyelitis, diphtheria, tetanus, pertussis and measles. Since its establishment, the EPI's list has been extended, and today it includes vaccines that have been developed since EPI has been established. Among these new vaccines, five are routinely being recommended by WHO for inclusion in all countries. These include hepatitis B (HepB) recombinant vaccine, haemophilus influenzae type $b$ conjugate vaccine (HibCV), pneumococcal conjugate vaccine (PCV), rotavirus vaccines (RotaV) and Human papilloma virus vaccine (HPV). While over the past four decades, globally there have been significant improvements in the performance of the national immunization programs (NIP), VPDs are unfortunately still responsible for a quarter of child deaths. Of major concern is that roughly half of these childhood deaths are reported from SSA. Especially, pneumonia and rotavirus diarrhea are the two leading causes of death among children under the age of five in SSA. The introduction of vaccines against these two diseases is steadily expanding across the region, today only six and four of the 10 countries in SSA have introduced the PCV and rotavirus vaccines respectively. The PCV-13 and monovalent rotavirus (RV1) are the vaccines most widely in use inthe NIP. The nations of SSA have succeeded in introducing HepB and HibCV vaccines into their NIP. Although the perinatal transmission of HBV contributes to the burden of hepatitis related diseases in SSA, the majority of the infants in SSA still do not receive the birth dose of HepB vaccine. This is partly due to the challenges the administration of the birth dose of HepB vaccine faces in SSA where still many babies are born at home. Understanding these administrative challenges of immunization better in the SSA settings and the progress made with new device technologies should help to significantly improve the number of infants receiving a HepB dose within 24hours of their life.

In addition to the burden of childhood, women in SSA are particularly at risk of cervical cancer. The region of SSA accounts approximately for a quarter of all cases and deaths related to HPV reported worldwide Even though it is proven that the HPV vaccination in adolescent girls is effective the HPV vaccine has not yet been largely introduced into the NIP in SSA. The HPV vaccine has reached young girls in Rwandan and South African in school-based programs and it is now in demonstration projects in the rest of the region. However the implementation of HPV vaccination in countries with lower school enrolment and attendance will still be a challenge.

The childhood immunization schedule in SSA is mainly based on the WHO standardized vaccination schedule established for the original EPI vaccines and is thus similar to immunization schedules in Europe. Having a harmonized childhood immunization schedule in a region such as SSA have advantages such as assuring mobility of citizens in the region, coalition of nations in negotiating lower vaccines prices with the manufacturers, reduction of the need of local clinical trials, facilitate the collection surveillance of data and increase the vaccine coverage.

Immunization has a great impact on the efforts made to meet the Millennium Development Goals (MDGs). Particularly, the fourth MDG which is to reduce child mortality, but also other MDGs such MDGs-1 and MDG-2. The introduction of new vaccines against the leading killer diseases of children under five (pneumonia and diarrhea) contribute to the decline of deaths in the nations of SSA. Based on current available data on pre and post vaccination for the SSA countries that introduced vaccines for the first time, it is quite reasonable to conclude that the introduction of new vaccines has resulted in great reduction of disease burden in these countries. Although there is a significant decline of burden of disease, it is essential that surveillance continues in the countries that introduced the new vaccines to determine whether the effective control persists or a booster dose becomes necessary. Despite the fact that the introduction of new vaccines in general does not affect the existing vaccine coverage (DTP-3), we observed a variation on the vaccine coverage in the NIP of countries with previously lower vaccine coverage. An in depth and multivariable analysis study will address to this finding.

Even though progress has been made in SSA, the region is still behind compared to developed countries. When a vaccine is first licensed in a developed country, it can take up to 20-30years before all people in developing countries have access to the vaccine. As VPDs disable and kill many African children every year, there is a great need to reduce this time span and accelerate and scale up the introduction of new vaccines into NIP of countries of SSA. However, as some countries do not have adequate capacity to handle challenges related to implementation of new interventions it is therefore crucial to understand better the decision-making process for NVI. While planning the introduction of new vaccines, decision makers should more proactively address the various factors such as (storage, dosing, stability, health workers) that can negatively impact on the immunization program as well as the health systems.

The immunization programs in SSA remain heavily donor dependent. There is not yet a clear African ownership initiative ready to overcome external donor dependence in the region. Based on the PAHO success story in Latin America on financial immunization, there is an urgent need for SSA countries to join forces and develop strategies and plans for the long-term financial sustainability of their immunization programs.

Due to the biological nature of vaccines, there are no generic vaccine products as such, which is reflected in the high entry costs to manufacturers. Vaccines and drugs thus have different market conditions. Not a long time ago, the majority of SSA could not afford to purchase the newer vaccines, whereas today, many SSA countries have an opportunity for huge discounts on vaccines through the publicprivate partnership (the GAVI Alliance). With support from the GAVI Alliance, substantial progress has been made in almost all countries of SSA for the introduction of new vaccines since 2000. Not only is 
the availability of funds behind the success of NVI. Countries with political stability, government effectiveness, regulatory quality, control of corruption and accountability are more likely to attract foreign aid and will therefore be able to benefit from these vaccines. The scaling up of the cold chain system and the vaccine logistics, improvement of reporting procedures and the training of health workforce will also be enhanced with NVI, though the basic infrastructure being in place will still be a pre-requisite. The launch of new vaccines in SSA often involves the high top national officials such as the Head of State and the African First Ladies. NVI may therefore also be an opportunistic instrument for political leaders in many SSA countries.

Strengths and limitations of the study: The strength of this study lies within the geographic region considered, the quality of articles and reports reviewed and its clear analytical framework. For the NVI in countries of SSA, (except south Sudan as new country) there were no limitations in terms of country-selection, all 48 countries of SSA have been considered in this review. Possible limitations might be the use of the data abstracted from reports or online database. As some studies done in the past questioned the validity of such data suggesting a significant level of over-reporting, limited quality of these data could indeed affect the validity of our results. ${ }^{128}$ In addition, while searching for reports involving human subjects, published or unpublished documentation written in English. It might happen to miss a paper that has been published in a language other than English as not all the countries of SSA use English as official language. These factors could influence the results of this paper, however only slightly so.

\section{Acknowledgments}

None.

\section{Conflicts of interest}

Author declares there are no conflicts of interest.

\section{Funding}

None.

\section{References}

1. Susan L, Plotkin SA. A short history of vaccination. Vaccines, $\left(6^{\text {th }}\right.$ edn $)$, Saunders, Philadelphia, USA. 2013. p.1-15

2. World health Organisation (WHO). Immunization. World health Organisation, Geneva. 2015.

3. Corcoran B. Global and National Trends in Vaccine Preventable Diseases. 2013.

4. Rappuoli R, Mandl CW, Black S, et al. Vaccines for the twenty-first century society. Nat Rev Immunol. 2012;11(12):11865-11872.

5. Poland G, Barrett A. The Old and the New: successful vaccines of the 20th century and approaches to making vaccines for the important diseases of the $21^{\text {st }}$ century. Curr Opin Immunol. 2010;21(3):305-307.

6. Keja K, Chan C, Hayden G, et al. Expanded programme on immunization World Health Stat. 1988;41(2):59-63.

7. World Health organization. National Immunization Programme (NIP) Module 5: Vaccines safety institutions and mechanisms. 2015.

8. World Health organization. Expanded Programme on Immunization(EPI). 2011.

9. World Health organization. National programmes and systems. 2014.

10. World Health organization. The Expanded Programme on Immunization: Benefits of immunization. 2013.
11. JAMISON DT, FEACHEM RG, MAKGOBA MW, et al. Disease and Mortality in Sub-Saharan Africa. (2nd edn), Washington DC, USA. 2006.

12. World health Organization (WHO). Expanded Programme on Immunization: global overview. 1985.

13. Department-Health. Epidemiological comments. Pretoria, South Africa. 2009.

14. Blumberg B. The Hepatitis B Virus Story Looking for One Thing, Finding Another. B Informed - Spring 2001 - Hepatitis B Foundation. 2001. p.9

15. Von Gottberg A, Cohen C, Whitelaw A, et al. Invasive disease due to Haemophilus influenzae serotype $\mathrm{b}$ ten years after routine vaccination, South Africa, 2003-2009. Vaccine. 2012;30(3):565-571.

16. Godal T. $30^{\text {th }}$ World Economic Forum (January 2000): GAVI Board Meeting Executive Summary. Davos, Switzerland. 2000.

17. Arevshatiana L, Clementsb Cj, Lwangac Sk, et al. An evaluation of infant immunization in Africa : is a transformation in progress ? Bulletin of the World Health Organization. 2007;85(6):421-500.

18. World Health organization. Global Immunization Vision and Strategy. Immunization, Vaccines and Biologicals. 2011.

19. World Health organization. Resolutions and decisions: sixty-fifth World Health Assembly. Geneva, Switzerland. 2012. p.21-26.

20. World Health organization. Global vaccine action plan 2011-2020. Immunization, Vaccines and biologics, Geneva, Switzerland. 2011. p.5-6.

21. Levine OS, Bloom DE, Cherian T, et al. The future of immunisation policy, implementation, and financing. Lancet. 2011;378(978):439-448.

22. World Health organization. First WHO seminar on Expansion of the use of immunization in developping countries. Kumasi-Ghana12-19 November 1974, Geneva, Switzerland. 1975. p.1-31.

23. Bele O, Barakamfitiye D. EPI in the WHO African region: current situation and constraints. John Libbey Eurotext. 1994;4(3):137-142.

24. World Health Organization. Table 3: Recommendations for Interrupted or Delayed Routine Immunization Summary of WHO Position Papers. 2015. p.1-9.

25. World Health Organization. Vaccine Preventable Diseases Monitoring System 2015 global summary. 2015.

26. Machingaidze S, Wiysonge CS, Hussey GD. Strengthening the expanded programme on immunization in Africa: looking beyond 2015. Plos med. 2013;10(3):e1001405.

27. Deroeck D, Wang SA. WHO-Principles and considerations for adding a vaccine to a national immunization programme. WHO Library Cataloguing-in-Publication Data, Geneva, Switzerland. 2014. p.1-2.

28. World Health Organization. Rubella vaccines: WHO position paper Wkly epidemiol rec . 2011;86(29):301-316.

29. Plotkin SA. The History of Rubella and Rubella Vaccination Leading to Elimination. Clin Infect Dis. 2006;1(43 Suppl 3):S164-S168.

30. World Health Organization. Country Planning Cycle Database/Africa. 2014.

31. IVAC. VIMS Report : Global Vaccine Introduction. A report on current global access to new childhood vaccines, Baltimore, USA. 2014. p.1-37.

32. Path. Country introductions of rotavirus vaccines. 2015.

33. Madhi SA, Bamford L, Ngcobo N. Effectiveness of pneumococcal conjugate vaccine and rotavirus vaccine introduction into the South African public immunisation programme. S Afr Med J. 2014;104(3 suppl 1):228-234. 
34. GAVI. Countries approved for support. 2015.

35. Binagwaho A, Ngabo F, Wagner CM, et al. Integration of comprehensive women's health programmes into health systems: cervical cancer prevention, care and control in Rwanda. Bull World Health Organ. 2013;91(9):697-703.

36. Perlman S, Wamai RG, Bain PA, et al. Knowledge and awareness of HPV vaccine and acceptability to vaccinate in sub-Saharan Africa: a systematic review. Plos one . 2014;9(3):e90912.

37. ICO HPV Information Centre. Human Papillomavirus and Related Diseases Report. Barcelona, Spain. 2015. p.1-245.

38. Wigle J, Coast E, Watson-Jones D. Human papillomavirus (HPV) vaccine implementation in low and middle-income countries (lmics): health system experiences and prospects. Vaccine. 2013;31(37):3811-3817.

39. USAID. Office of Gender Equality \& Women's Empowerment: Gender Statistics. US Agency for International Development. 2014.

40. Unicef. Education. Rwanda, Africa. 2014.

41. Binagwaho A, Wagner CM, Gatera M, et al. Achieving high coverage in Rwanda's national human papillomavirus vaccination programme. Bull World Health Organ. 2012;90(8):623-628.

42. Grant Mj, Hallman Kk. Pregnancy-related School Dropout and Prior School Performance in KwaZulu-Natal, South Africa. Stud Fam Plann. 2006;39(4):369-382.

43. World Health Organization. New Vaccine Post-Introduction Evaluation (PIE) Tool. Immunization vaccine Biologicals, Geneva, Switzerland. 2010. p.1-65.

44. CDC. Progress in Introduction of Pneumococcal Conjugate Vaccine Worldwide, 2000-2012. Weekly. 2013;62(16):308-311.

45. World Health Organization. Vaccine-preventable diseases: monitoring system. 2015 global summary. 2015

46. World Health Organization. Hepatitis B vaccines. Wkly epidemiol rec 2009;84(40):405-419.

47. World Health Organization. Hepatitis B Wkly Epidemiol Rec. 2009;84(40):405.

48. World Health Organization. Haemophilus Influenza type b (hib) vaccination-Position paper-July 2013. Wkly Epidemiol Rec. 2013; 88(39):413-426.

49. World Health Organization (WHO) Pneumococcal disease. Internationa travel and health.

50. World Health Organization. Pneumococcal vaccines WHO position paper-2012. Wkly Epidemiol Rec. 2012;87(14):129-144.

51. Hajjeh R. Accelerating introduction of new vaccines: barriers to introduction and lessons learned from the recent Haemophilus influenzae type B vaccine experience. Philos Trans $R$ Soc Lond B Biol Sci. 2011;366(1579):2827-2832.

52. Fortuin M, Chotard J, Jack AD, et al. Efficacy of hepatitis B vaccine in the Gambian expanded programme on immunisation. Lancet. 1993;341(8853):1129-1131.

53. Viviani S, Jack A, Hall AJ, et al. Hepatitis B vaccination in infancy in The Gambia: protection against carriage at 9 years of age. Vaccine 1999; 17(23-24):2946-2950.

54. Hall AJ, Roberston RL, Crivelli PE, et al. Cost-effectiveness of hepatitis B vaccine in The Gambia. Trans R Soc Trop Med Hyg. 87(3):333-336.

55. CDC. Hepatitis B. 2014. p.149-174.

56. GAVI. Haemophilus influenzae type b vaccine support. 2014.

57. Mulholland K, Hilton S, Adegbola R, et al. Randomised trial of Haemophilus influenzae type-b tetanus protein conjugate vaccine [corrected] for prevention of pneumonia and meningitis in Gambian infants. Lancet. 1997;349(9060):1191-1197.
58. Hussey GD, Lasser ML, Reekie WD. The costs and benefits of a vaccination programme for Haemophilus influenzae type B disease. $S$ Afr Med J. 1995;85(5):20-25.

59. Ward RL, Bernstein DI. Rotarix: a rotavirus vaccine for the world. Clin infect dis. 2009;48(2):222-228.

60. Berry SA, Johns B, Shih C, et al. The cost-effectiveness of rotavirus vaccination in Malawi. J infect dis. 2010;202 Suppl:S108-S115.

61. Madhi SA, Cunliffe NA, Steele D, et al. Effect of human rotavirus vaccine on severe diarrhea in African infants. $N$ Engl $J$ med. 2010;362(4):289-298.

62. Armah GE, Sow SO, Breiman RF, et al. Efficacy of pentavalent rotavirus vaccine against severe rotavirus gastroenteritis in infants in developing countries in sub-Saharan Africa: a randomised, double-blind, placebocontrolled trial. Lancet. 2010;376(9741):606-614.

63. Abbott C, Tiede B, Armah G, et al. Evaluation of cost-effectiveness of live oral pentavalent reassortant rotavirus vaccine introduction in Ghana. Vaccine. 2012;30(15): 2582-2587.

64. Klugman KP, Madhi SA, Huebner RE, et al. A trial of a 9-valent pneumococcal conjugate vaccine in children with and those without HIV infection. New Engl J med. 2003; 349(14):1341-1348.

65. Cutts FT, Zaman SM, Enwere G, et al. Efficacy of nine-valent pneumococcal conjugate vaccine against pneumonia and invasive pneumococcal disease in The Gambia: randomised, double-blind , placebo-controlled trial. Lancet . 2005;365(9465):1139-1146.

66. Kim SY, Lee G, Goldie SJ. Economic evaluation of pneumococcal conjugate vaccination in The Gambia. BMC infect dis. 2010;10:260.

67. Tina Khoie. PCV 13: Review of Pre-Licensure Safety Data. Pediatric Advisory Committee Meeting, US Food and Drug Administration (FDA). 2012.

68. Choi EH, Kim KH, Kim YJ, et al. Recommendation for use of the newly introduced pneumococcal protein conjugate vaccines in Korea. Korean J pediatr. 2011;54(4):146-151

69. Hammitt LL, Akech DO, Morpeth SC, et al. Population effect of 10 -valent pneumococcal conjugate vaccine on nasopharyngeal carriage of Streptococcus pneumoniae and non-typeable Haemophilus influenzae in Kilifi, Kenya: findings from cross-sectional carriage studies. Lancet Global Health. 2014;2(7):e397-e405.

70. Hawkes D, Lea CE, Berryman MJ. Answering human papillomavirus vaccine concerns; a matter of science and time. Infect agent cancer $2013 ; 8(1): 22$.

71. Dochez C, Bogers JJ, Verhelst R, et al. HPV vaccines to prevent cervical cancer and genital warts: an update. Vaccine. 2014;32(14):1595-1601.

72. Cutts FT, Franceschi S, Goldie S, et al. Human papillomavirus and HPV vaccines : a review. Bull World Health Organ. 2007;85(9):719-726.

73. Mantel C, Wang SA. The privilege and responsibility of having choices: decision-making for new vaccines in developing countries. Health policy plan. 2012;27(Suppl 2):ii1-ii4.

74. Gordon WS, Jones A, Wecker J. Introducing multiple vaccines in low- and lower-middle-income countries: issues, opportunities and challenges. Health policy plan. 2012; 27(Suppl 2):ii17-ii26.

75. SIVAC. NITAG Resource Center. 2014.

76. World Health Organization. National advisory committees on immunization. Immunization, Vaccines and Biologicals. 2014

77. Joseph M. Vaccine Licensure: African Perspective. J VISR/VISP Meeting, National Institutes of Health, USA. 2013

78. Burchett HE, Mounier-Jack S, Griffiths UK, et al. New vaccine adoption qualitative study of national decision-making processes in seven lowand middle-income countries. Health policy plan. 2012;27:ii5-ii16. 
79. Saxenian H, Hecht R, Kaddar M, et al. Overcoming challenges to sustainable immunization financing: early experiences from GAVI graduating countries. Health policy plan. 2015;30(2):197-205.

80. World Health Organization. The second African Regional Conference on Immunization (ARCI). Ouagadougou, Burkina Faso, Africa. 2010.

81. World Health Organization. WHO position statement on Hib conjugate vaccines. Wkly Epidemiol Rec. 2004;79:173.

82. World Health Organization. WHO position paper on Hib conjugate vaccines. Wkly Epidemiol Rec. 2006;81:445-452.

83. Shearer JC, Stack ML, Richmond MR, et al. Accelerating policy decisions to adopt haemophilus influenzae type B vaccine: a global, multivariable analysis. Plos med. 2010;7(3):e1000249.

84. Glatman-Freedman A, Cohen ML, Nichols KA, et al. Factors affecting the introduction of new vaccines to poor nations: a comparative study of the Haemophilus influenzae type B and hepatitis B vaccines. Plos one. 2010;5(11):e13802.

85. Gambia proposals, reports \& plans, GAVI The vaccine Alliance, Gambia, Africa.

86. Unicef. Launch of pneumococcal vaccine initiative aims to protect thousands in Kenya (2012). Unicef, Kenya. 2012.

87. Liberia begins protecting its children against pneumococcal disease. Unicef, West and Central Africa. 2014.

88. World Health Organization. Uganda introduces pneumococcal vaccine to curb infant and child deaths. World Health Organization, Africa 2013

89. Ininihazwe DC. Burundi to cut child mortality rates. East-Africa Business Week. 2014.

90. WHO-Afro. Malawi introduces the Rotavirus vaccine to reduce diarrhea illnesses and deaths among children, World Health Organization, Africa 2012.

91. Fadera H. VP Njie-Saidy launches new vaccine. Observer Dalily, Gambia, Africa. 2009.

92. Tall F. Two long-awaited new vaccines will protect our children in Burkina Faso from pneumonia and diarrhea. DEFEATDD. 2013.

93. Nchewnang-Ngassa BY. Rotavirus Diarrhoea: Cameroon's First Lady Leads Vaccination Crew. Yallafrica, Cameroon, Africa. 2014

94. Unicef. DR Congo introduces new vaccine against one of its leading causes of child death. 2011.

95. WHO-Afro. Ghana introduces 2 new vaccines; 2nd African Vaccination Week launched. 2012.

96. Musoni E. First Lady leads campaign against Cervical Cancer. The New Times, Rwanda. 2011

97. SANAC. Minister of health launches HPV vaccination campaign. 2014

98. SABAHI. Tanzania to vaccinate children against pneumonia, diarrhea 2012.

99. WHO-Afro. The United Republic of Tanzania commemorates world immunization week with a call to ensure all children are vaccinated 2013

100. Mwesigye S. Pre-teen girls targeted in cervical cancer fight. The Observer. 2012

101. Bohn L. Tackling Cancer with a Dab of Vinegar. The daily beast. 2013

102. Chauke-Moagi BE, Mumba M. New vaccine introduction in the East and Southern African sub-region of the WHO African region in the context of GIVS and mdgs. Vaccine. 2012;30(Suppl 3):C3-8.
103. Bingham A, Drake JK, LaMontagne DS. Sociocultural issues in the introduction of human papillomavirus vaccine in low-resource settings. Arch Pediatr Adolesc med. 2009;163(5):455-461.

104. USAID-JSI. Immunization Programs for Healthy Children. Immunization basics. 2009.

105. Tagbo BN, Mwenda JM, Armah G, et al. Epidemiology of rotavirus diarrhea among children younger than 5 years in Enugu, South East, Nigeria. Pediatr infect dis $J$. 2014;33(Suppl 1):S19-S22.

106. Wiese-Posselt M, Reiter S, Gilsdorf A, et al. Needs and obstacles of uniform immunisation schedules in the European Union. Bundesgesundheitsblatt Gesundheitsforschung Gesundheitsschutz. 2009;52(11):1099-1104

107. European-Commission. Report on a Workshop of Vaccination Strategy 13-14 February 2008. Luxembourg, Europe. 2008.

108. Theeten H, Nohynek H, Coenen TM, et al. EuSANH workshop "Reasons behind the differences in national vaccination schedules for under-five", European Public Health pre-conference workshop, Malta, 8 November 2012. Vaccine. 2013;31(42):4694-4696.

109. Mihigo R, Nshimirimana D, Hall A, et al. Control of viral hepatitis infection in Africa: are we dreaming? Vaccine. 2013;31(2):341-346.

110. Baker L, Amayeza Mp. The face of South Africa's Expanded Programme on Immunisation (EPI) schedule. SA Pharmaceutical Journal. 2010; $18-21$.

111. Candotti D, Danso K, Allain JP. Materno fetal transmission of hepatitis B virus genotype $\mathrm{E}$ in Ghana, west Africa. $J$ Gen virol. 2007;88(10):2686-2695.

112. Kew MC. Progress towards the comprehensive control of hepatitis B in Africa: a view from South Africa. Gut. 1996;38 (Suppl 2):S31-S36.

113. Kramvis A, Clements CJ. Implementing a birth dose of hepatitis $\mathrm{B}$ vaccine for home deliveries in Africa--too soon? Vaccine. 2010;28(39):6408-6410.

114. MSF. Civil society groups call on GAVI to support birth dose vaccination for hepatitis B. 2014

115. GAVI. Hepatitis B vaccine at birth - GAVI responds to MSF. 2014.

116. Howell J, Lemoine M, Thursz M. Prevention of materno-foetal transmission of hepatitis B in sub-Saharan Africa: the evidence, current practice and future challenges. $J$ viral hepat. 2014;21(6):381-396.

117. Kinfu Y, Dal Poz MR, Mercer H, et al. The health worker shortage in Africa : are enough physicians and nurses being trained ? Bullet World Health Organ. 2014;87(3):225-230.

118. UNICEF The urgent need to address the health worker crisis in Africa p. 108

119. Mahoney RT, Maynard JE. The introduction of new vaccines into developing countries. Vaccine. 1999;17(7-8):646-652.

120. Outreville JF. Foreign direct investment in the health care sector and most-favoured locations in developing countries. Eur J Health Econ. 2007;8(4):305-312.

121. Duclos P, Okwo-Bele JM, Gacic-Dobo M, et al. Global immunization: status, progress, challenges and future. BMC int health human rights. 2009;9(Suppl 1):S2

122. Mary Robinson. Immunization is all about human rights. Chicago Tribune. 2005

123. PAHO. Introduction of New Vaccines and Underutilized. Recent Advances in Immunization. 2006. p.121-122.

124. Paterson $\mathrm{P}$, Larson $\mathrm{Hj}$. The role of publics in the introduction of new vaccines. Health policy plan. 2012;27(Suppl 2):ii77-ii79. 
125. Wonodi CB, Privor-Dumm L, Aina M, et al. Using social network analysis to examine the decision-making process on new vaccine introduction in Nigeria. Health policy and planning. 2012;27(Suppl 2):ii27-ii38.

126. Chan M. Beyond expectations: 40 years of EPI. Lancet. 2014;383(9930):1697-1698.
127. PAHO. PAHO Revolving Fund. 2014.

128. Lim SS1, Stein DB, Charrow A, et al. Tracking progress towards universal childhood immunisation and the impact of global initiatives: a systematic analysis of three-dose diphtheria, tetanus, and pertussis immunisation coverage. Lancet. 2008;372(9655):2031-2046. 